Article

\title{
Automatic Supervisory Controller for Deadlock Control in Reconfigurable Manufacturing Systems with Dynamic Changes
}

\author{
Husam Kaid ${ }^{1, *(1)}$, Abdulrahman Al-Ahmari ${ }^{1}$, Zhiwu Li ${ }^{2,3}$ and Reggie Davidrajuh ${ }^{4}$ (1) \\ 1 Department of Industrial Engineering, College of Engineering, King Saud University, \\ Riyadh 11421, Saudi Arabia; alahmari@ksu.edu.sa \\ 2 Institute of Systems Engineering, Macau University of Science and Technology, Taipa, Macau 999078, China; \\ systemscontrol@gmail.com \\ 3 School of Electro-Mechanical Engineering, Xidian University, Xi'an 710071, China \\ 4 Department of Electrical Engineering and Computer Science, Faculty of Science and Technology, \\ University of Stavanger, 4036 Stavanger, Norway; reggie.davidrajuh@uis.no \\ * Correspondence: yemenhussam@yahoo.com
}

Received: 10 July 2020; Accepted: 27 July 2020; Published: 30 July 2020

\begin{abstract}
In reconfigurable manufacturing systems (RMSs), the architecture of a system can be modified during its operation. This reconfiguration can be caused by many motivations: processing rework and failures, adding new products, adding new machines, etc. In RMSs, sharing of resources may lead to deadlocks, and some operations can therefore remain incomplete. The objective of this article is to develop a novel two-step solution for quick and accurate reconfiguration of supervisory controllers for deadlock control in RMSs with dynamic changes. In the first step, the net rewriting system (NRS) is used to design a reconfigurable Petri net model under dynamic configurations. The obtained model guarantees boundedness behavioral property but may lose the other properties of a Petri net model (i.e., liveness and reversibility). The second step develops an automatic deadlock prevention policy for the reconfigurable Petri net using the siphon control method based on a place invariant to solve the deadlock problem with dynamic structure changes in RMSs and achieve liveness and reversibility behavioral properties for the system. The proposed approach is tested using examples in the literature and the results highlight the ability of the automatic deadlock prevention policy to adapt to RMSs configuration changes.
\end{abstract}

Keywords: reconfigurable manufacturing system; Petri net; deadlock; siphon; supervisory controller

\section{Introduction}

A typical example of discrete event systems is an automated manufacturing system (AMS) [1,2]. It enables various product types to be entered at discrete times by sharing resources like machines, automatic controlled vehicles, automated tools, robots, and buffers at asynchronous or simultaneous operations. AMSs have to cope with unexpected and rapid market changes on a competitive global market. They must make rapid modifications to their software and hardware to meet these dynamic changes. This requirement cannot, however, be satisfied successfully with traditional automated manufacturing systems, which require large capital investments. Reconfigurable manufacturing systems have now been developed to deal with those drawbacks in traditional automated manufacturing systems [3-5]. Reconfigurable manufacturing systems are a new kind of production systems that are randomly and dynamically configured in real time. Such configurations involve processing rework and failures, adding new products and machines, and adding new handling device. In RMSs, a set of system resources can be used to process each component according to a specific process sequence. 
This sharing of resources, however, may lead to deadlocks, and some operations can therefore remain incomplete. Therefore, dealing with deadlock problem is critical for RMSs.

Petri nets (PNs) are widely used for the scheduling, deadlock analysis and control in AMSs as graphical and mathematical modelling tools [6-14]. They can be used to describe characteristics and behaviors of AMSs such as synchronization, concurrency, conflict, causal dependence, and sequencing. Petri nets can be used for behavioral features, for example boundedness and liveness [15,16]. From a technical point of view, several policies based on Petri nets have been proposed. These policies are based on three strategies: (i) deadlock detection and recovery, (ii) deadlock avoidance, and (iii) deadlock prevention [15,17]. Most of these policies have proposed deadlock control in Petri nets through structural analysis $[6,18]$ and reachability graph analysis [19-21]. In addition, three criteria to evaluate and construct an AMS supervisor have been proposed, namely behavioral permissiveness, computational complexity, and structural complexity [15,22].

Recently, several approaches have been adapted to deal with dynamic changes in manufacturing systems [7,23-36]. They primarily concentrate in two directions: direct and indirect. Direct approaches provide modification mechanisms or particular rules for system structure configurations, while indirect approaches typically import additional mechanisms for system reconfiguration specifications. The event-condition-action (ECA) paradigm is developed by Almeida et al. [30] for the design of reconfigurable logic controllers. Their research has demonstrated that the reconfiguration process is highly dependent on the modularity level of the logical control system and that not all "modular" structures can be reconfigured. For a class of discrete event systems (DESs), Sampath et al. [26] presented a reconfiguration approach for their control specifications, subject to linear constraint. This approach is suited to systems such as hospital management systems and can be reconfigured in non-real time. In order to evaluate and improve the performance of the control architecture, Dumitrache et al. [27] developed a real-time reconfigurable supervised control architecture for large manufacturing systems. A model-based control design for reconfigurable manufacturing systems is developed by Ohashi and Shin [28] through state transition diagrams and general graph representation taking into account configuration and reuse of design data. Kalita and Khargonekar [29] introduced a hierarchical structure and a framework for modeling, analysis, specification, and design of logic controllers for RMSs, which allows rapid reconfigurability and reusability of the controller during reconfiguration. In [23], reconfigurable manufacturing systems were used to replace the existing manufacturing systems to offer higher convertibility and flexibility such as dedicated production systems. Serial and parallel configurations, a rules-based matrix approach has been developed and implemented. In addition, a higher-level deadlock control method is presented for the serial and parallel configurations.

Net Rewriting Systems (NRS) are another graph-based reconfiguration mechanism [34]. In terms of pattern matching and dynamic structure replacements, the reconfiguration occurs. By the implementation of a Turing machine the expressive power was shown to be Turing equivalent. A subset of net rewriting systems, called reconfigurable nets, have also been provided with an algorithm to flatten a Petri net to standard. This subset only restricts NRS to those transformations that remain unchanged in the number of places and transitions, that is, only the flow relation can be changed. Flattening significantly increases the size of transitions by multiplying the number of reconfigurations by the amount of transitions. The NRS is used in logic controllers with improved net rewriting systems [35]. The improved NRS version restricts the rewriting rules to ensure important structural characteristics such as boundedness, liveness, and reversibility are not invalidated. In addition, in [24], an improved net rewriting system (INRS) was developed with the aim of reconfiguring an RMS supervisory controller based on PNs. Changes to an RMS modification were made to rewrite rules that were then applied in the initial PN controller. The INRS is first proposed as a reconfiguration basis. The structure of a Petri net model can be changed dynamically. Then, the study provided three representations of the RMS modification and suggested an INRS-based method to the design of the Petri net controller of an RMS. In this approach, the properties of behavioral, i.e., the boundedness, reversibility, and liveness of a modified system, were not verified or validated. 
In [31], colored timed PNs (CTPN) were used in the modelling of RMSs and a mechanism to describe reconfigurability in the CTPN architecture was introduced that leads to a new architecture supporting the reconfiguration. This mechanism includes reconfigurable transitions, specific places, and inhibitor arcs. Wu and Zhou introduced intelligent token Petri net (ITPN) [25]. In their model, tokens representing job instances carry real-time knowledge about system states and changes, just like intelligent cards in practice such that dynamical changes of a system can be easily modeled. These formalisms can describe the reconfiguration behavior of the system. However, some of dynamic changes do not clearly define the modularity, which brings confusion to engineers in designing, understanding, and future redevelopment. Correctness of the system such as coherence of states before and after system reconfigurations is not considered. In addition, temporal constraints, which are of great significance in real-time systems are not mentioned. In [32], reconfigurable object nets (RONs) are used to model, simulate, and analyze RMSs. A formal method was proposed for fulfilling a new production requirement. The configuration consists of new extrusion and cutting machines. The reconfiguration is represented as graph transformations, RON tool was used to simulate the reconfigured systems and TINA [37] and PIPE [38] software tools were used to carry out the analysis.

The work of Silva et al. [36] explored the principles of the different approaches and takes from them the best practices. Configuration mechanisms were proposed using Holonic and multiagent system methods to allow a reconfigurable distributed production control system to systematically detect faults. To describe communication interfaces, the principle of service-oriented architecture was used. Hybrid top-down and bottom-up approaches were presented using Petri net models. In [33], object-oriented Petri nets (ORPNs) and $\pi$-calculus were used as two complementary formalisms. Initial RMSs structure and system behavior were modeled by ORPN while the $\pi$-calculus was used to describe RMSs' reconfiguration. To evaluate, check, and validate RMSs, Petri nets and $\pi$-calculus supporting tools were used. The reconfigurability mechanism and consistency of RMSs could be analyzed by $\pi$-calculus. In [7], a new model is proposed, namely the intelligent colored token Petri net (ICTPN), which simulated dynamic configurations of systems such as adding new machines, processing failures and rework, machine failures, processing routes changes, removing old machines, and adding new products. The primary idea is that smart colored tokens were part types which represented real-time knowledge of system status and configurations. This allowed for the effective modeling of dynamic system configurations. The proposed ICTPN could modularly model dynamic system changes to generate a very compact model. Moreover, when configurations appear, only the colored token of the part type, which is changed from the current model was changed. The resulting ICTPN model ensures that the behavioral properties such as deadlock-free, conservative, and reversible were guaranteed.

All of the above methods with PNs attempted to deal with dynamic configuration issues in manufacturing systems. However, most of them do not include an algorithm or mechanism for reconfiguration, could not guarantee the properties of behavioral Petri net (i.e., boundedness (or safeness), liveness, and reversibility), or could not ensure that the results of the reconfiguration are correct, accurate or valid. In addition, few techniques for rapid and valid reconfiguration of literature deadlock control supervisors were presented.

The objective of this article is to develop a novel two-step solution for quick and accurate reconfiguration of supervisory controllers for deadlock control in RMSs with dynamic changes. In the first step, the net rewriting system used in $[34,39]$ was adapted to design a reconfigurable Petri net model under dynamic configurations. The obtained model guarantees boundedness behavioral property but may lose the other properties of a Petri net model (i.e., liveness and reversibility). This means that the reconfigured Petri net model has finite states, deadlocks, and does not behave cyclically. For this issue, the second step develops an automatic deadlock prevention policy for reconfigurable Petri net using the siphon control method based on place invariant to solve the deadlock problem with dynamic structure changes in RMSs and achieve liveness and reversibility behavioral properties for the system. Thus, the developed approach has the ability of adapting to RMS configuration changes.

The major applications of the developed approach are as follows: 
1. Mass customization manufacturing can use the proposed approach to address its difficulties. For example, by trying to make products available rapidly to consumers, a high quality production of a wide variety of products can be maintained and achieve low costs in line with standard products.

2. Lean productivity concept can also use the proposed approach to enable a company to implement an RMS in order to improve the exploitation of the part of the resources for various family products and to minimize waste from the idle resource of an RMS.

3. Agile manufacturing can use the proposed approach to facilitate rapid products changeovers, rapid introduction of new products and unattended operation.

4. Flexible manufacturing systems can use the proposed approach to increase response to a variety of customers and markets. Moreover, scalability to the desired volume of products and convertibility to current systems, machines, robots, and controls are increased in accordance with the new production requirements.

This paper is organized as follows. Section 2 describes basic concepts of Petri nets, reconfigurable Petri nets. Section 3 presents the deadlock prevention policy for reconfigurable Petri net based on the concept of minimal siphons and place invariants. The behavioral and quantitative analysis of the proposed reconfigurable Petri net are presented in Section 4. A real-world case study is presented in Section 5 to demonstrate the application of the proposed approach. Conclusions and future research are presented in Section 6.

\section{Preliminaries}

\section{1. $S^{3} P R N E T$}

Definition 1. A simple sequential process $\left(S^{2} P\right)$ is a Petri net model with $N=\left(\left\{p^{0}\right\} \cup P_{A}, T, F\right)$ if (1) $N$ is a strongly connected state machine and (2) each circuit $N$ contains place $p^{0}$, where $p^{0}$ is a process idle place, $P_{A}=\left\{p_{1}, p_{2}, \ldots, p_{m}\right\}$ is a set of operation places, $T=\left\{t_{1}, t_{2}, \ldots, t_{n}\right\}$ is a set of transitions, $P_{B}=P_{A} \cup\left\{p^{0}\right\}, P_{B}$ $\cap T=\emptyset, P_{B} \cup T \neq \emptyset$, and $F:\left(P_{B} \times T\right) \cup\left(T \times P_{B}\right) \rightarrow I N$ is a set of weighted arcs called flow relations, where IN $=\{0,1,2, \ldots\}$.

Definition 2. A simple sequential process with resources $\left(S^{2} P R\right)$ is a Petri net model with $N=\left(\left\{p^{0}\right\} \cup P_{A} \cup\right.$ $\left.P_{R}, T, F\right)$ if

1. the subnet created by $Y=P_{A} \cup\left\{p^{0}\right\} \cup T$ is an $S^{2} P$;

2. $P_{R} \neq \emptyset$ and $\left(P_{A} \cup\left\{p^{0}\right\}\right) \cap P_{R}=\emptyset$, where $P_{R}$ is called a set of resource places;

3. $P_{C}=P_{A} \cup\left\{p^{0}\right\} \cup P_{R}, F \subseteq\left(P_{C} \times T\right) \cup\left(T \times P_{C}\right)$ is flow relations;

4. $\quad \bullet\left(p^{0}\right) \cap P_{R}=\left(p^{0}\right) \bullet \bullet P_{R} \neq \emptyset$;

5. $\forall p \in P_{A}, \forall t \in \bullet p, \forall t^{\prime} \in p^{\bullet}, \exists r_{p} \in P_{R}, \bullet t \cap P_{R}=t^{\bullet} \cap P_{R}=\left\{r_{p}\right\} ;$

6. $\forall r \in P_{R}, \bullet^{\bullet} r \cap P_{A}=r^{\bullet \bullet} \cap P_{A} \neq \emptyset$ and $\bullet r \cap r^{\bullet} \neq \emptyset$;

Definition 3. Let $N=\left(\left\{p^{0}\right\} \cup P_{A} \cup P_{R}, T, F\right)$ be an $S^{2} P R$ with $M_{o}$ being an initial marking of net $N$. An $S^{2} P R$ is called acceptably marked if (1) $M_{o}\left(p^{0}\right) \geq 1$, (2) $M_{o}(p)=0, \forall p \in P_{A}$, and (3) $M_{o}(r) \geq 1, \forall r \in P_{R}$.

Recursively, a system of $S^{2} P R$ is called an $S^{3} P R$.

Definition 4. A system of $S^{2} P R, S^{3} P R$, is defined recursively as follows:

1. An $S^{2} P R$ is an $S^{3} P R$;

1. Let $N_{i}=\left(\left\{p_{i}^{0}\right\} \cup P_{A i} \cup P_{R i}, T_{i}, F_{i}\right), i=\{1,2\}$, be two $S^{3} P R s$ such that $\left(\left\{p^{0}{ }_{1}\right\} \cup P_{A 1}\right) \cap\left(\left\{p^{0}{ }_{2}\right\} \cup P_{A 2}\right)=\emptyset$, $P_{R 1} \cap P_{R 2}=P_{D}, P_{A 1} \cap P_{A 2} \neq P_{D}$, and $T_{1} \cap T_{2} \neq \emptyset$; then, the net $N=\left(\left\{p^{0}\right\} \cup P_{A} \cup P_{R}, T, F\right)$ is an $S^{3} P R$ resulting from the integration of $N_{1}$ and $N_{2}$ by the set of common $P_{D}$ (denoted as $N_{1} \circ N_{2}$ ) and expressed as: (1) $p^{0}=\left\{p_{1}^{0}\right\} \cup\left\{p_{2}^{0}\right\}$, (2) $P_{A}=P_{A 1} \cup P_{A 2}$, (3) $P_{R}=P_{R 1} \cup P_{R 2}$, (4) $T=T_{1} \cup T_{2}$, and (5) $F=F_{1} \cup F_{2}$. 
The integration of $n S^{2} P R N_{1}-N_{n}$ via $P_{D}$ is expressed by $\otimes_{i=1}^{n} N_{i} . \overline{N_{i}}$ is used to indicate the $S^{2} P$ from which the $S^{2} P R N_{i}$ is built.

Definition 5. Let $N_{i}=\left(\left\{p_{i}^{0}\right\} \cup P_{A i} \cup P_{R i}, T_{i}, F_{i}\right), i=\{1,2\}$, be two $S^{3} P R s . M_{o}$ is an initial marking of $N$. (N, $\left.M_{o}\right)$ is called acceptably marked if $(1)\left(N, M_{0}\right)$ is an acceptably marked $S^{2} P R$, and (2) $N=N_{1} \circ N_{2}$, where $\left(N_{i}\right.$, $\left.M_{i 0}\right)$ is called an acceptably marked $S^{3} P R$ and

1. $\forall i \in\{1,2\}, \forall p \in P_{A i} \cup\left\{p^{0}{ }_{i}\right\}, M_{o}(p)=M_{i o}(p)$.

2. $\forall i \in\{1,2\}, \forall r \in P_{R i} \backslash P_{D}, M_{o}(r)=M_{i o}(r)$.

3. $\forall i \in\{1,2\}, \forall r \in P_{D}, M_{o}(p)=\max \left\{M_{10}(r), M_{2 o}(r)\right\}$.

Definition 6. Let $N=\left(\left\{p^{0}\right\} \cup P_{A} \cup P_{R}, T, F, W, M_{o}\right)$ be an $S^{3} P R$, where $W:\left(P_{C} \times T\right) \cup\left(T \times P_{C}\right) \rightarrow I N$ is a mapping that assigns a weight to an arc and $M_{0}: P_{C} \rightarrow I N$ is the initial marking.

Definition 7. Let $N=\left(\left\{p^{0}\right\} \cup P_{A} \cup P_{R}, T, F, W, M_{0}\right)$ be an $S^{3} P R$. $N$ is said to be an ordinary net if $p \in P_{C}, t \in$ $T, \forall(p, t) \in F$, and $W(p, t)=1$.

Definition 8. Let $N=\left(\left\{p^{0}\right\} \cup P_{A} \cup P_{R}, T, F, W, M_{0}\right)$ be an $S^{3} P R$. $N$ is said to be a weighted net if $\exists p \in P_{C}, \exists t$ $\in T,(p, t) \in F$, and $W(p, t)>1$.

Definition 9. Let $N=\left(\left\{p^{0}\right\} \cup P_{A} \cup P_{R}, T, F, W, M_{0}\right)$ be an $S^{3} P R$, where $p$ and $t$ are a place and a transition in $N$, respectively. The preset (postset) of $p$ is the set of all input (output) transitions of $p$, i.e., $\cdot p=\{t \in T \mid(t, p) \in$ $F\}\left(p^{\bullet}=\{t \in T \mid(p, t) \in F\}\right.$ ). The preset (postset) of $t$ is the set of all input (output) places of $t$, i.e., $\bullet^{\bullet} t=\left\{p \in P_{C} \mid\right.$ $(p, t) \in F\}\left(t^{\bullet}=\left\{p \in P_{C} \mid(t, p) \in F\right\}\right)$.

Definition 10. Let $N=\left(\left\{p^{0}\right\} \cup P_{A} \cup P_{R}, T, F, W, M_{0}\right)$ be an $S^{3} P R$. $N$ is self-loop free if for all $p, t \in P_{C} \cup T$; $W(p, t)>0$ implies $W(t, p)=0$ and has a self-loop if $W(t, p)>0$.

Definition 11. Let $N=\left(\left\{p^{0}\right\} \cup P_{A} \cup P_{R}, T, F, W, M_{o}\right)$ be an $S^{3} P R$ and $M$ be a marking of $N$, where $M$ is a mapping $M: P_{C} \rightarrow I N$ and the pth element of $M$, expressed by $M(p)$, is the number of tokens in place $p$.

Definition 12. Let $N=\left(\left\{p^{0}\right\} \cup P_{A} \cup P_{R}, T, F, W, M_{0}\right)$ be an $S^{3} P R$. A transition $t \in T$ is enabled if $\forall p \in \bullet^{\bullet}$, $M(p) \geq W(p, t)$.

Definition 13. Let $N=\left(\left\{p^{0}\right\} \cup P_{A} \cup P_{R}, T, F, W, M_{0}\right)$ be an $S^{3} P R$. The marking $M^{\prime}$ resulting from the firing of an enabled transition $t \in T$ at marking $M$ is denoted by $M[t\rangle M^{\prime}$ and expressed as follows:

$$
M^{\prime}(p)=\left[\begin{array}{ll}
M(p)+W(p, t) & \text { if } p \in \bullet \backslash t \bullet \\
M(p)-W(t, p) & \text { if } p \in t^{\bullet} \backslash \bullet t \\
M(p)+W(t, p)-W(p, t) & \text { if } p \in t^{\bullet} \cap \bullet t \\
M(p) & \text { otherwise }
\end{array}\right]
$$

Definition 14. Let $N=\left(\left\{p^{0}\right\} \cup P_{A} \cup P_{R}, T, F, W, M_{o}\right)$ be an $S^{3} P R . R(N, M)$ is a set of reachable markings from $M$ in $N$, which is expressed by nodes and arcs; nodes represent markings that are labeled with $M_{i}$ and arcs represent transition firings that are labeled with $t$. If $t$ fires, then there is an arc from marking $M_{i}$ to marking $M_{j}$ and $M_{j}$ is reached.

Definition 15. Let $N=\left(\left\{p^{0}\right\} \cup P_{A} \cup P_{R}, T, F, W, M_{0}\right)$ be an $S^{3} P R$. A transition $t \in T$ is live at $M_{o}$ if $\forall M \in$ $R\left(N, M_{0}\right), \exists M^{\prime} \in R(N, M)$ such that $M^{\prime}[t\rangle$ holds. $\left(N, M_{0}\right)$ is dead at $M_{o}$ if there does not exist $t \in T$ such that $M_{o}[t\rangle$ holds. $\left(N, M_{0}\right)$ is weakly live or live-locked if $\forall M \in R\left(N, M_{0}\right), \exists t \in T, M[t\rangle$ holds. $\left(N, M_{0}\right)$ is quasi-live if $\forall t \in T, \exists M \in R\left(N, M_{0}\right)$ such that $M[t\rangle$ holds. 
Definition 16. Let $N=\left(\left\{p^{0}\right\} \cup P_{A} \cup P_{R}, T, F, W, M_{0}\right)$ be an $S^{3} P R$. [N] is said to be the incidence matrix of net $N$, where $[N]$ is a $|P| \times|T|$ integer matrix with $[N](p, t)=W(t, p)-W(p, t)$. For a place $p$ (transition $t$ ), its incidence vector, a row (column) in $[N]$, is expressed as $[N](p,).([N](., t)$.

Definition 17. Let $N=\left(\left\{p^{0}\right\} \cup P_{A} \cup P_{R}, T, F, W, M_{0}\right)$ be an $S^{3} P R$. A marking $M^{\prime}$ is called reachable from $M$ if there exists a sequence of transitions $\delta=t_{1} t_{2} t_{3} \ldots t_{n}$ that can be fired, and markings $M_{1}, M_{2}, M_{3}, \ldots$, and $M_{n-1}$ are such that $M\left[t_{0}\right\rangle M_{1}\left[t_{1}\right\rangle M_{2}\left[t_{2}\right\rangle M_{3} \ldots M_{n}\left[t_{n}\right\rangle M^{\prime}$ holds, expressed as $M[\delta\rangle M^{\prime}$, satisfies the state equation $M^{\prime}=M+[N] \vec{\delta} \cdot \vec{\delta}: T \rightarrow I N$ is called a firing count vector or a Parikh vector that maps $t$ in $T$ to the number of occurrences of $t$ in $\delta$.

Definition 18. Let $N=\left(\left\{p^{0}\right\} \cup P_{A} \cup P_{R}, T, F, W, M_{0}\right)$ be an $S^{3} P R . N$ is said to be bounded if there exists $q \in I N, \forall M \in R\left(N, M_{0}\right), \forall p \in P_{C}, M(p) \leq q$. $\left(N, M_{0}\right)$ is structurally bounded if it is bounded for any $M_{0}$.

Definition 19. LLet $N=\left(\left\{p^{0}\right\} \cup P_{A} \cup P_{R}, T, F, W, M_{o}\right)$ be an $S^{3} P R$. $N$ is called safe if $\forall M \in R\left(N, M_{0}\right), \forall p \in$ $P_{C}, M(p) \leq 1$. (N, $\left.M_{0}\right)$ is $q$-safe if it is $q$-bounded.L

Consider the example of AMS illustrated in Figure 1a. The system has one robot R1 and one machine M1. Machine M1 processes one part at a time and robot R1 holds one part at a time. There are buffers for loading/unloading. Furthermore, one part type is considered to be processed in the system. The part operation sequence is illustrated in Figure 1b. Figure 2 shows the $S^{3} P R$ net of the AMS example. It has six places and four transitions. The following sets of places can be used: $P^{0}=\left\{p_{1}\right\}$, $P_{R}=\left\{p_{5}, p_{6}\right\}$, and $P_{A}=\left\{p_{2}, p_{3}, p_{4}\right\}$. There are five reachable markings on the Petri model. The initial marking is $M_{o}=(5,0,0,0,1,1)^{\mathrm{T}}$, which represents the different raw parts that are to be processed synchronously within the system, including preconditions, input signals, buffers and resource status, such as machines and robot. Places are generally used to represent the resource status, operations, and activities. The transitions are used to express control changes from one state to another. Directed arcs correspond to the material, resource, information flow, and control flow direction between states. Material, information, and resources are represented by tokens.

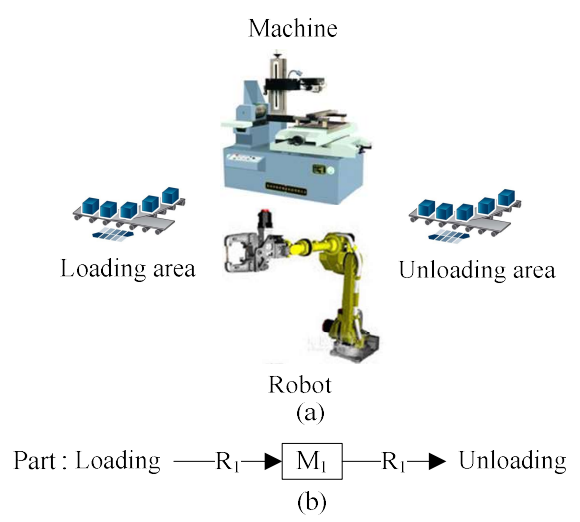

Figure 1. (a) Automated manufacturing system (AMS) example and (b) operation sequence. 


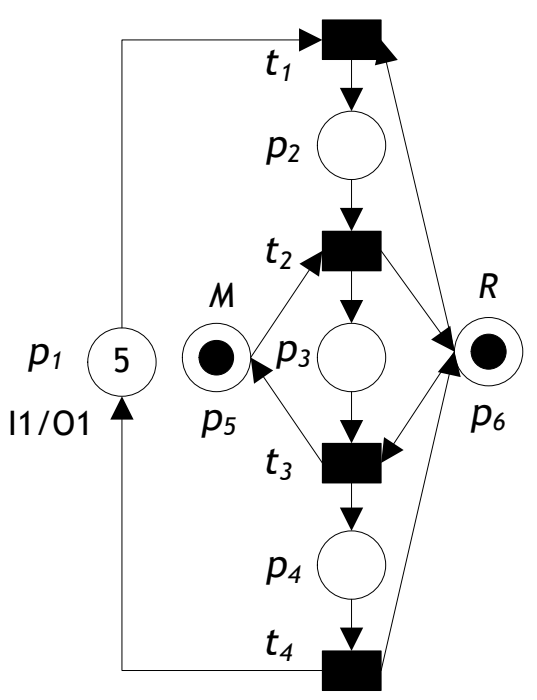

Figure 2. A system of $\left(S^{2} \mathrm{P}\right)$ (simple sequential process) $\left(\mathrm{S}^{3} \mathrm{PR}\right)$ net of the AMS.

\subsection{Reconfigurable $S^{3} P R$ Net}

This section presents definitions and theorems in the reconfigurable $S^{3} P R$ nets, which are originally proposed by $[34,35,39]$.

Definition 20. Let $N=\left(\left\{p^{0}\right\} \cup P_{A} \cup P_{R}, T, F, W, M_{0}, K\right)$ be a finite-capacity $S^{3} P R$, where $p^{0}, P_{A}, P_{R}, T, F, W$, and $M_{0}$ are defined in Definitions 1-6. K: $P_{C} \rightarrow I N$ is the function of capacity that assigns to each place $p$ the maximal number of tokens $K(p)$.

Definition 21. Let $\left(N_{i}, M_{i}\right)$ be two $S^{3} P R$ nets with $N_{i}=\left(P_{C i}, T_{i}, F_{i}, W_{i}, M_{i}, K_{i}\right), i=1,2 . N_{1}$ and $N_{2}$ are called morphism nets if there exists a bijection $\Psi: N_{1} \rightarrow N_{2}, \Psi=\left(\Psi_{P C}: P_{C 1} \rightarrow P_{C 2}, \Psi_{T}: T_{1} \rightarrow T_{2}\right)$ such that for all a, $b \in P_{C 1} \cup T_{1}, F_{1}(a, b) \in N_{1}=F_{2}(\Psi(a), \Psi(b)) \in N_{2}$, and for all $p \in P_{C 1}, M_{1}(p) \leq M_{2}\left(\Psi_{P C}(p)\right)$.

Definition 22. Let $\left(N_{i}, M_{i}\right)$ be two $S^{3} P R$ nets with $N_{i}=\left(P_{C i}, T_{i}, F_{i}, W_{i}, M_{i}, K_{i}\right), i=1,2 . N_{1}$ is called the full subnet of $N_{2}$ if there exists an injection function that maps places to places and transitions to transitions, denoted by $\xi: N_{1} \rightarrow N_{2}, \xi\left(P_{C 1}\right) \subseteq P_{C 2}$, and $\xi\left(T_{1}\right) \subseteq T_{2}$ such that for all $a, b \in P_{C 1} \cup T_{1}, F_{1}(a, b)=F_{2}(\xi(a), \xi(b))$.

In the algebraic, a rewriting rule is a transformation approach that can change and combine the Petri nets dynamically. The main idea is to define and change the system configurations as a graph rewriting rule.

Definition 23. Let $N_{R}$ be a reconfigurable $S^{3} P R$ with $N_{R}=\left(\left(N, M_{0}\right), \mathcal{R}\right)$, where $\left(N, M_{o}\right)$ is an $S^{3} P R$ net with $N=\left(P_{C}, T, F, W, M_{0}, K\right)$ and $\mathcal{R}=\left\{r r_{1}, r r_{2}, r r_{3}, \ldots, r r_{m}\right\}$ is called a set of rewriting rules or dynamic configurations if

1. For all $r r \in \mathcal{R}, r r=\left\{L, R, \varphi, \bullet \varphi, \varphi \varphi^{\bullet}\right.$;

2. $L=\left(P_{C L}, T_{L}, F_{L}, W_{L}, M_{o L}, K_{L}\right)$ is called the left-hand side;

3. $R=\left(P_{C R}, T_{R}, F_{R}, W_{R}, M_{o R}, K_{R}\right)$ is called the right-hand side;

4. $\varphi \subseteq\left(P_{C L} \times P_{C R}\right) \cup\left(T_{L} \times T_{R}\right)$ is said to be an interface transfer relation of $r$ that relates places of $L$ to places of $R$ and transitions of $L$ to transitions of $R, P_{C L} \varphi \subseteq P_{C R}, \varphi P_{C R} \subseteq P_{C L}, T_{L} \varphi \subseteq T_{R}$, and $\varphi T_{R} \subseteq P_{L}$;

5. $\bullet \varphi \subseteq \varphi$ is said to be an input interface transfer relation, expressed as $\bullet \varphi=\left\{\left(\left\{L . p_{i}\right\},\left\{\right.\right.\right.$ R. $\left.\left.\left.p_{i}\right\}\right)\right\}$ or $\left\{\left(\left\{L . t_{i}\right\},\{R\right.\right.$. $\left.\left.\left.t_{i}\right\}\right)\right\}$, and L.* or R.* means to input nodes "*" in Lor R;

6. $\varphi^{\bullet} \subseteq \varphi$ is named output interface transfer relation, $\varphi^{\bullet}=\left\{\left(\left\{L . p_{j}\right\},\left\{R . p_{j}\right\}\right)\right\}$ or $\left\{\left(\left\{L . t_{j}\right\},\left\{\right.\right.\right.$ R.t $\left.\left.\left.t_{j}\right\}\right)\right\}$, and $L^{*} .^{*}$ or $R^{*}$ means to output nodes "*" in L or $R$;

7. for all $r r_{i}, r r_{j} \in \mathcal{R}(i \neq j), \xi\left(L_{i}\right) \cap \xi\left(L_{j}\right) \neq \emptyset$, a rewriting must be guaranteed without overlap; moreover, the order of $r r_{i}, r r_{j}$ does not impact the result of the rewriting. 
Definition 24. Let $N_{R}$ be a reconfigurable $S^{3} P R$ with $N_{R}=\left(\left(N, M_{0}\right), \mathcal{R}\right)$. A new rewriting reconfigurable net $N_{R}$ is an $S^{3} P R$ net $\left(N_{R}, M_{R}\right)$ with $N_{R}=\left(P_{C}, T, F, W, M_{R}, K\right)$, and a net $\left(N, M_{o}\right)$ is called the initial state of the rewriting net model.

Definition 25. Let $N_{R}$ be a reconfigurable $S^{3} P R$ with $N_{R}=\left(\left(N, M_{0}\right), \mathcal{R}\right)$. A state graph in $N_{R}$ is a labeled directed graph whose nodes are the marking of $N_{R}$, expressed as:

1. Transition firing: If Arcs labeled with $t$ can fire in the net $\left(N_{1}, M_{1}\right)$, leading to $\left(N_{2}, M_{2}\right):\left(N_{1}, M_{1}\right) \stackrel{t}{\rightarrow}\left(N_{2}\right.$, $\left.M_{2}\right) \Leftrightarrow\left(N_{1}=N_{2}\right.$ and $M_{1}\left[t_{2}\right\rangle M_{2}$ in $\left.N_{1}\right)$.

2. Configuration changing: Arcs labeled with $r=\{L, R, \varphi, \bullet \varphi, \varphi \cdot\}$ from state $\left(N_{1}, M_{1}\right)$ to state $\left(N_{2}, M_{2}\right)$ if there is $\xi: L \rightarrow N_{1}$ so that, $\forall a \notin \xi(L)$ and $b \in L$ if

2.1. $a \in \bullet \xi(b) \Rightarrow b \in \bullet \varphi$ and $a \in \xi(b)^{\bullet} \Rightarrow b \in \varphi^{\bullet}$.

2.2. $N_{1}=\left(P_{C 1}, T_{1}, F_{1}, W_{1}, M_{1}, K_{1}\right)$ and $N_{2}=\left(P_{C 2}, T_{2}, F_{2}, W_{2}, M_{2}, K_{2}\right)$ holds the following: $P_{C 2}=P_{C 1}-\xi\left(P_{C 1 L}\right)+P_{C 1 R}$ and $T_{2}=T_{1}-\xi\left(T_{1 L}\right)+T_{1 R}$. Note that $-(+)$ means deleting(inserting) places or transitions from (to) $N_{1}$ and the places name of $P_{C 1 R}$ and $T_{1 R}$ inserted into $N_{1}$ must be different to prevent clashes.

Definition 26. Let $N_{R}$ be a reconfigurable $S^{3} P R$ with $N_{R}=\left(\left(N, M_{0}\right), \mathcal{R}\right)$. Let $N_{1}$ and $N_{2}$ be two states in $N_{R}$ with $N_{1}=\left(P_{C 1}, T_{1}, F_{1}, W_{1}, M_{10}, K_{1}\right)$ and $N_{2}=\left(P_{C 2}, T_{2}, F_{2}, W_{2}, M_{20}, K_{2}\right)$. A net $N_{1}$ is the restriction of $a$ net $N_{2}$ if $P_{C 1} \subseteq P_{C 2}, T_{1} \subseteq T_{2}$, and $F_{1}=F_{2} \cap\left(\left(P_{C 1} \times T_{1}\right) \cup\left(T_{1} \times P_{C 1}\right)\right)$ and expressed by $N_{1} \subseteq N_{2}$.

Definition 27. Let $N_{R}$ be a reconfigurable $S^{3} P R$ with $N_{R}=\left(\left(N, M_{0}\right), \mathcal{R}\right)$. Let $N_{1}$ and $N_{2}$ be two states in $N_{R}$ with $N_{1}=\left(P_{C 1}, T_{1}, F_{1}, W_{1}, M_{10}, K_{1}\right)$ and $N_{2}=\left(P_{C 2}, T_{2}, F_{2}, W_{2}, M_{20}, K_{2}\right)$. The set of weighted arcs (flow relation) $F_{2}$ is expressed as:

$$
F_{2}(a, b)=\left[\begin{array}{ll}
F_{1}(a, b) & \text { if } a \notin R \wedge b \notin R \\
F_{R}(a, b) & \text { if } a \in R \wedge b \in R \\
\sum_{b_{i} \in \bullet \bullet b} F_{1}\left(a, \xi\left(y_{i}\right)\right) & \text { if } a \notin R \wedge b \in R \\
\sum_{a_{i} \in \varphi \bullet a} F_{1}\left(\xi\left(a_{i}\right), b\right) & \text { if } a \in R \wedge b \notin R
\end{array}\right]
$$

Definition 28. Let $N_{R}$ be a reconfigurable $S^{3} P R$ with $N_{R}=\left(\left(N, M_{o}\right), \mathcal{R}\right)$. Let $N_{1}$ and $N_{2}$ be two states in $N_{R}$ with $N_{1}=\left(P_{C 1}, T_{1}, F_{1}, W_{1}, M_{10}, K_{1}\right)$ and $N_{2}=\left(P_{C 2}, T_{2}, F_{2}, W_{2}, M_{20}, K_{2}\right)$. The marking of $M^{\prime}(p), p \in P_{C 2}$, is expressed as:

$$
M^{\prime}(p)=\left[\begin{array}{ll}
M(p) & \text { if } p \notin R \\
\sum_{p^{\prime} \in \varphi p} M\left(\xi\left(p^{\prime}\right)\right) & \text { if } p \in R
\end{array}\right]
$$

Theorem 1. Let $N_{R}$ be a reconfigurable $S^{3} P R$ with $N_{R}=\left(\left(N, M_{0}\right)\right.$, R). Let $N_{1}$ and $N_{2}$ be two states in $N_{R}$ with $N_{1}=\left(P_{C 1}, T_{1}, F_{1}, W_{1}, M_{10}, K_{1}\right)$ and $N_{2}=\left(P_{C 2}, T_{2}, F_{2}, W_{2}, M_{2 o}, K_{2}\right), P_{C 1}, T_{1} \neq \emptyset$ and $\mathcal{R}=\{r r\}, r r=\{L, R, \varphi$, $\bullet \varphi, \varphi \cdot\}$. If $L$ and $R$ are a single place or single transition, then the obtained $N_{2}$ by rr is equal to $N_{1}$.

Proof. Straightforward.

Theorem 2. Let $N_{R}$ be a reconfigurable $S^{3} P R$ with $N_{R}=\left(\left(N, M_{0}\right), \mathcal{R}\right)$. Let $N_{1}$ and $N_{2}$ be two states in $N_{R}$ with $N_{1}=\left(P_{C 1}, T_{1}, F_{1}, W_{1}, M_{10}, K_{1}\right)$ and $N_{2}=\left(P_{C 2}, T_{2}, F_{2}, W_{2}, M_{20}, K_{2}\right), P_{C 1}, T_{1} \neq \emptyset$ and $\mathcal{R}=\{r r\}, r r=\{L, R, \varphi$, $\bullet \varphi, \varphi \bullet$. If $\left(N_{1}, M_{1}\right)$ is bounded, $L$ is a single place or single transition and $R$ is an $S^{3} P R$ net, then the resulting $\left(N_{2}, M_{2 o}\right)$ net by $r r$ is bounded. 
Proof. The rewriting of $N_{2}$ using $r r$ is similar to replacing a place/transition by the $S^{3} P R$ net. Therefore, the boundedness can be established by checking if the $\mathrm{S}^{3} \mathrm{PR}$ net is well constructed and behaved. The resulting net $\left(N_{2}, M_{20}\right)$ maintains the boundedness because the $S^{3} P R$ net is well constructed and behaved.

Corollary 1. Let $N_{R}$ be a reconfigurable $S^{3} P R$ with $N_{R}=\left(\left(N, M_{0}\right), \mathcal{R}\right)$. Let $N_{1}$ and $N_{2}$ be two states in $N_{R}$ with $N_{1}=\left(P_{C 1}, T_{1}, F_{1}, W_{1}, M_{10}, K_{1}\right)$ and $N_{2}=\left(P_{C 2}, T_{2}, F_{2}, W_{2}, M_{20}, K_{2}\right), P_{C 1}, T_{1} \neq \emptyset$ and $\mathcal{R}=\{r r\}, r r=\{L$, $R, \varphi, \bullet^{\bullet} \varphi, \varphi \cdot$. If $\left(N_{1}, M_{10}\right)$ is bounded, $L$ is an $S^{3} P R$ Petri net and $R$ is a single place or single transition, then the resulting net $\left(\mathrm{N}_{2}, M_{20}\right)$ by rr is bounded.

Corollary 2. An $S^{3} P R$ net $\left(N_{2}, M_{20}\right)$ can be a bounded net and a full subnet of $\left(N_{1}, M_{10}\right)$.

Theorem 3. Let $N_{R}$ be a reconfigurable $S^{3} P R$ with $N_{R}=\left(\left(N, M_{0}\right)\right.$, R). Let $N_{1}$ and $N_{2}$ be two states in $N_{R}$ with $N_{1}=\left(P_{C 1}, T_{1}, F_{1}, W_{1}, M_{10}, K_{1}\right)$ and $N_{2}=\left(P_{C 2}, T_{2}, F_{2}, W_{2}, M_{2 o}, K_{2}\right), P_{C 1}, T_{1} \neq \emptyset$ and $\mathcal{R}=\{r r\}, r r=\{L, R, \varphi$, $\bullet \varphi, \varphi \cdot\}$. If $\left(N_{1}, M_{10}\right)$ is bounded, $L$ is an $S^{3} P R$ net and $R$ is an $S^{3} P R$ net, then the resulting net $\left(N_{2}, M_{20}\right)$ by rr is bounded.

Proof. The rewriting of $\mathrm{N}_{2}$ using $r r$ is similar to replacing an $\mathrm{S}^{3} \mathrm{PR}$ net by another $\mathrm{S}^{3} \mathrm{PR}$ net. Therefore, the boundedness can be established by checking if the $\mathrm{S}^{3} \mathrm{PR}$ net is well constructed and behaved. The resulting net $\left(N_{2}, M_{20}\right)$ maintains the boundedness because the $S^{3} P R$ net is well constructed and behaved.

Based on Definitions 20-28 and Theorems 1-3, the developed reconfiguration procedures for $\mathrm{S}^{3} \mathrm{PR}$ net algorithm are constructed as follows:

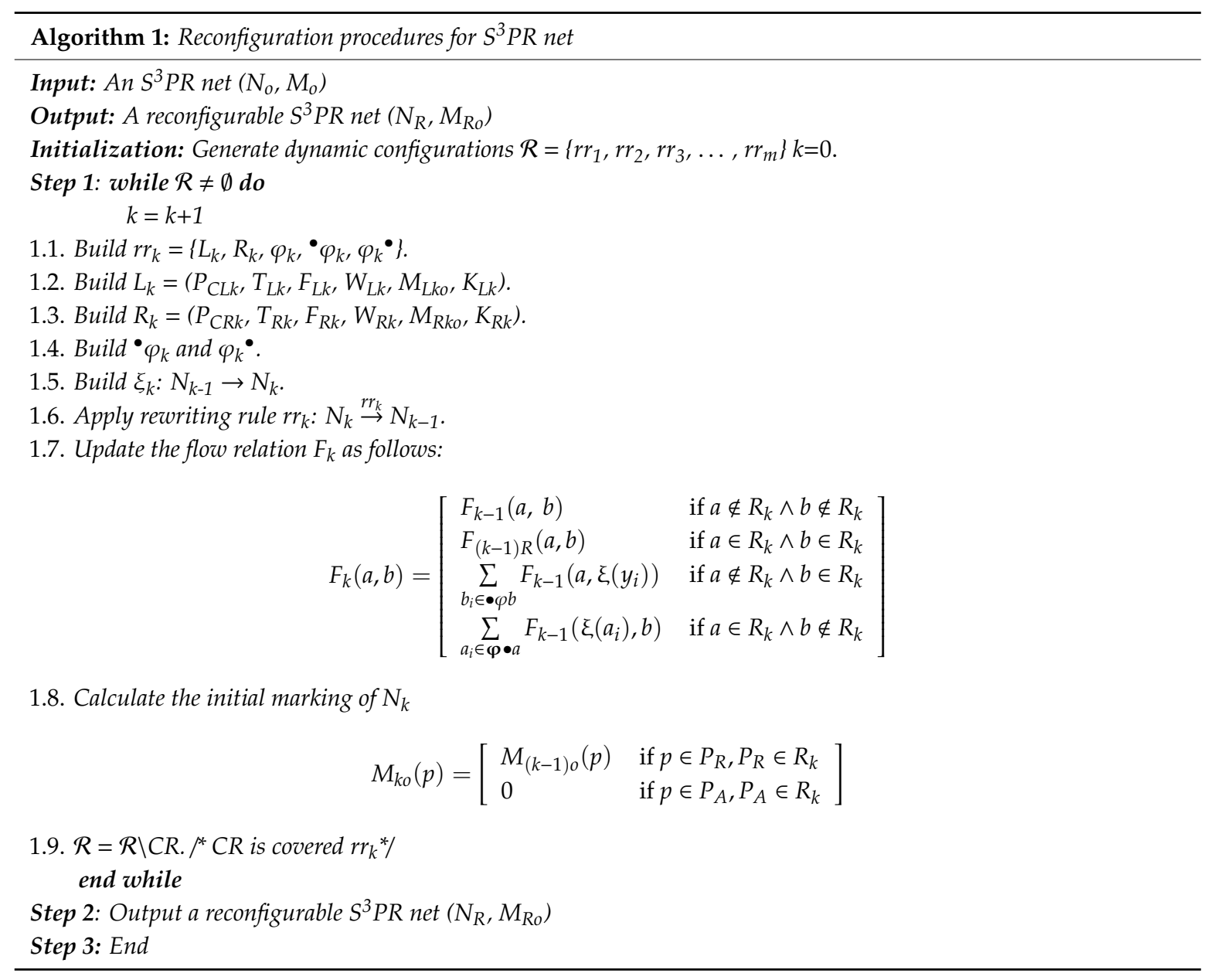


To illustrate the proposed Algorithm 1, reconsider the initial $S^{3} \mathrm{PR}$ net $\left(N_{o}, M_{o}\right)$ illustrated in Figure 2. Suppose that the first system configuration includes adding new machine. In this scenario, a new machine M2 is assigned to the system $\left(N_{o}, M_{o}\right)$ to process a part after $\mathrm{M}$, a robot is needed to load/unload a part to/from M2. To model the addition of new machine by using the synthesis procedure of Algorithm 1, we construct a configuration as a rewriting rule $\mathcal{R}=\left\{r r_{1}\right\}$ with $r r_{1}=\left\{L_{1}, R_{1}\right.$, $\varphi_{1}, \varphi_{1}, \varphi_{1} \bullet$, where $L_{1}$ and $R_{1}$ are illustrated in Figures $3 \mathrm{a}$ and $3 \mathrm{~b}$, respectively. We have $\xi_{1}: N_{1} \rightarrow$ $N_{0}, \varphi_{1}=\left(\left\{p_{1}, p_{6}, p_{7}, p_{8}, p_{9}\right\},\left\{t_{4}, t_{5}, t_{6}\right\}\right), \bullet^{\bullet} \varphi_{1}=\left(\left\{L_{1} . t_{4}\right\},\left\{R_{1} . t_{4}\right\}\right)$, and $\varphi_{1} \bullet=\left(\left\{L_{1} . p_{1}, L_{1} . p_{6}\right\},\left\{R_{1} . p_{1}\right\}\right)$. Then the obtained reconfigurable $S^{3} \mathrm{PR}$ net $\left(N_{1}, M_{10}\right)$ is illustrated in Figure $3 c$.

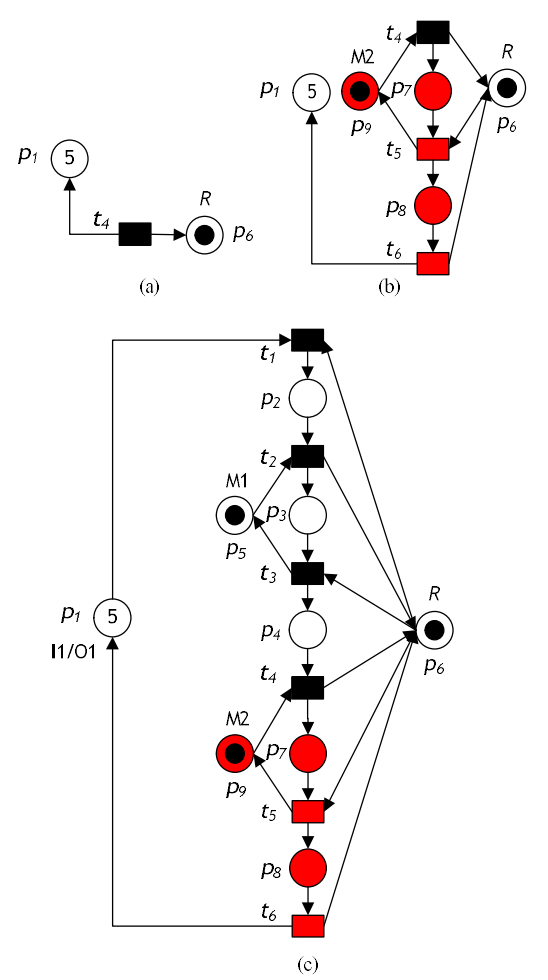

Figure 3. A reconfigured $S^{3} P R$ net by addition of new machine. (a) Left hand side net $L$. (b) Right hand side net $R$. (c) A reconfigurable $S^{3} P R$ net $\left(N_{1}, M_{10}\right)$.

The second configuration includes adding a new product. In this scenario, a new product (part B) is assigned to a system, which indicates that a new operation sequence is assigned and the system requires an adjustment to its Petri net model structure. To model the addition of new product by using the synthesis procedure of Algorithm 1, we constructed a configuration as a rewriting rule $\mathcal{R}=\left\{r r_{2}\right\}$ with $r r_{2}=\left\{L_{2}, R_{1}, \varphi_{2}, \varphi^{\bullet}, \varphi_{2} \bullet\right\}$, where $L_{2}$ and $R_{2}$ are illustrated in Figures $4 \mathrm{a}$ and $4 \mathrm{~b}$, respectively. We have $\xi_{2}: N_{2} \rightarrow N_{1}, \varphi_{2}=\left(\left\{p_{5}, p_{6}, p_{10}, p_{11}, p_{12}, p_{13}\right\},\left\{t_{7}, t_{8}, t_{9}, t_{10}\right\}\right), \bullet \varphi_{2}=\left(\left\{L_{2} \cdot p_{5}, L_{2} \cdot p_{6}\right\},\left\{R_{2} . t_{7}\right\}\right)$, and $\varphi_{2} \cdot=$ $\left(\left\{L_{2} \cdot p_{5}, L_{2} \cdot p_{6}\right\},\left\{R_{2} \cdot t_{10}\right\}\right)$. Then the obtained reconfigurable $S^{3} \mathrm{PR}$ net $\left(N_{2}, M_{20}\right)$ is illustrated in Figure 4c.

The third system configuration involves rework. In this scenario, a part can be inspected after all operations have been completed. The system can proceed on the basis of the original sequence of operation if the configuration is carried out properly. Otherwise, rework is needed. By using Algorithm 1, the production operations of the reworked part can be exactly and easily modeled by considering rework operations as alternative sequences. Reconsider the reconfigurable $S^{3} P R$ net $\left(N_{2}\right.$, $M_{2}$ ) illustrated in Figure 4c. Suppose that an inspection machine M3 is added to a system and that part B is processed in M1. Then, part B is moved to M3 by Robot 1 to check if there are defects in part B. If part B performed properly, then it will leave the system by Robot 1. Otherwise, if part B has defects, rework is needed, and part B is moved to M1 by Robot 1 . To model the rework operation by using the synthesis procedure of Algorithm 1, we construct a configuration as a rewriting rule $\mathcal{R}=\left\{r r_{3}\right\}$ with $r r_{3}=\left\{L_{3}, R_{3}, \varphi_{3}, \bullet^{\bullet} \varphi_{3}, \varphi_{3} \bullet\right\}$, where $L_{3}$ and $R_{3}$ are illustrated in Figure 5a,b, respectively. We have 
$\xi_{3}: N_{3} \rightarrow N_{2}, \varphi_{3}=\left(\left\{p_{5}, p_{6}, p_{10}, p_{11}, p_{12}, p_{13}, p_{14}, p_{15}, p_{16}, p_{17}\right\},\left\{t_{7}, t_{8}, t_{9}, t_{10}, t_{11}, t_{12}, t_{13}, t_{14}, t_{15}\right\}\right), \cdot \varphi_{3}=$ $\left(\left\{L_{3} . t_{7}\right\},\left\{R_{3} . t_{7}\right\}\right)$, and $\varphi_{3}{ }^{\bullet}=\left(\left\{L_{3} \cdot t_{10}\right\},\left\{R_{3} . t_{14}\right\}\right)$. Then the obtained reconfigurable $S^{3} \mathrm{PR}$ net $\left(N_{3}, M_{30}\right)$ is illustrated in Figure 5c.

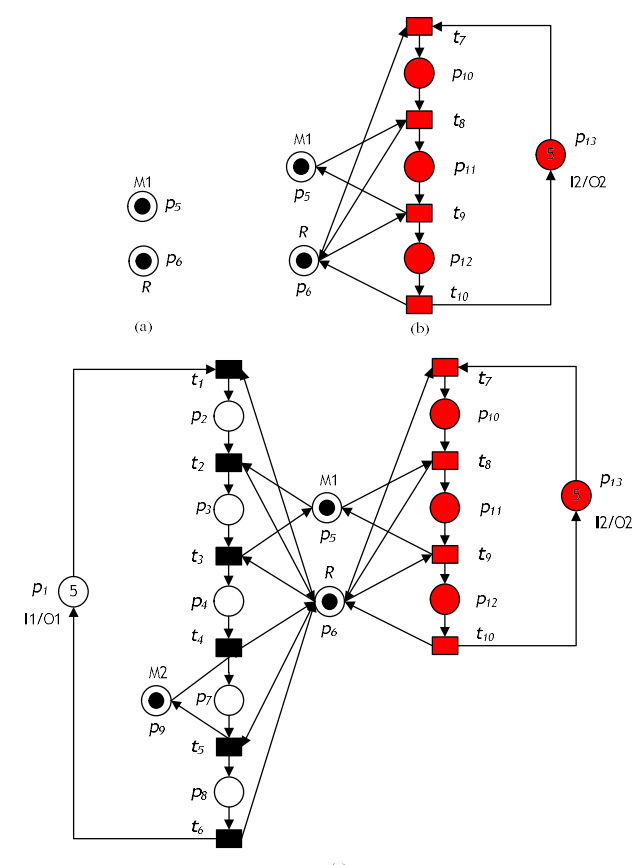

Figure 4. A reconfigured $S^{3} P R$ net by addition of new product. (a) Left hand side net $L$. (b) Right hand side net $R$. (c) A reconfigurable $S^{3} P R$ net $\left(N_{2}, M_{2 o}\right)$.
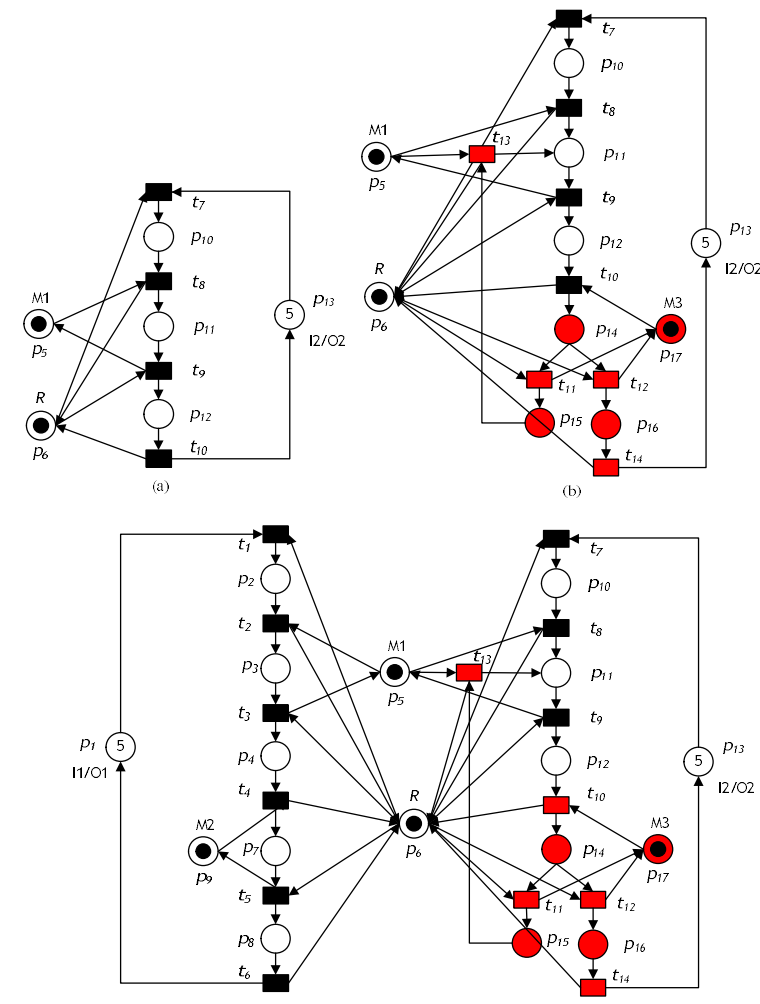

Figure 5. A reconfigured $S^{3} P R$ net by rework. (a) Left hand side net L. (b) Right hand side net R. (c) A reconfigurable $S^{3} \mathrm{PR}$ net $\left(\mathrm{N}_{3}, \mathrm{M}_{30}\right)$. 
Finally, a configuration includes adding a new robot. In this scenario, a new robot $\mathrm{R} 2$ is assigned to the system $\left(N_{3}, M_{30}\right)$ to load/unload a part A to/from M1 and M2. To model the addition of the new robot by using the synthesis procedure of Algorithm 1, we construct a configuration as a rewriting rule $\mathcal{R}=\left\{r r_{4}\right\}$ with $r r_{4}=\left\{L_{4}, R_{4}, \varphi_{4},{ }^{\bullet} \varphi_{4}, \varphi_{4} \bullet\right\}$, where $L_{4}$ and $R_{4}$ are illustrated in Figure 6a,b, respectively. We have $\xi_{4}: N_{4} \rightarrow N_{3}, \varphi_{4}=\left(\left\{p_{1}, p_{2}, p_{3}, p_{4}, p_{6-1}, p_{6} 2, p_{7}, p_{8}, p_{10}, p_{11}, p_{12}, p_{14}, p_{15}, p_{16}\right\},\left\{t_{1}, t_{2}, t_{3}, t_{4}, t_{5}, t_{6}\right.\right.$, $\left.\left.t_{7}, t_{8}, t_{9}, t_{10}, t_{11}, t_{12}, t_{13}, t_{14}, t_{15}\right\}\right), \cdot \varphi_{4}=\left(\left\{L_{4} \cdot t_{1}, L_{4} \cdot t_{7}\right\},\left\{R_{4} \cdot t_{1}, R_{4} \cdot t_{7}\right\}\right)$, and $\varphi_{4} \cdot=\left(\left\{L_{4} \cdot t_{6}, L_{4} \cdot t_{14}\right\},\left\{R_{4} \cdot t_{6}\right.\right.$, $\left.\left.R_{4} \cdot t_{14}\right\}\right)$. Then the obtained reconfigurable $S^{3} \mathrm{PR}$ net $\left(N_{4}, M_{40}\right)$ is illustrated in Figure $6 \mathrm{c}$.

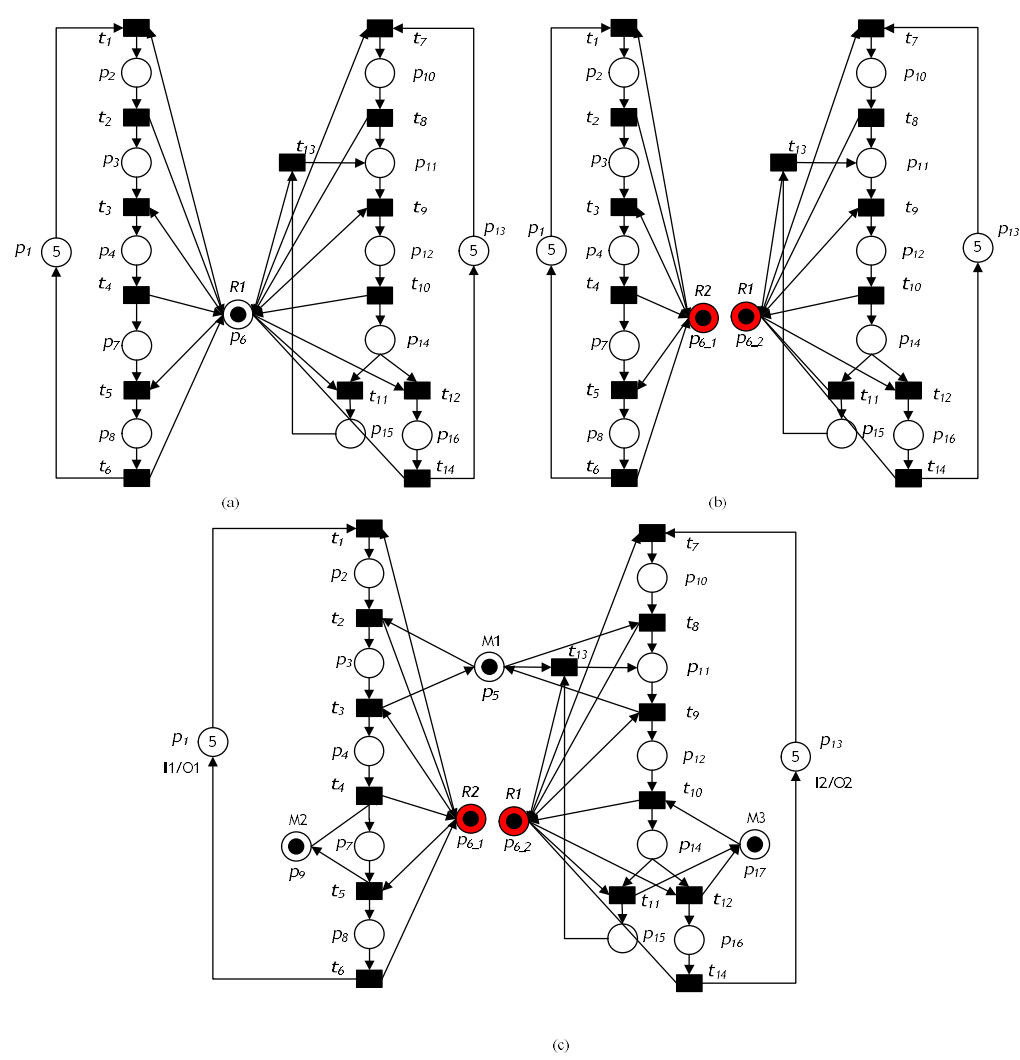

Figure 6. A reconfigured $S^{3} \mathrm{PR}$ net by addition of a new robot. (a) Left hand side net $L$. (b) Right hand side net $R$. (c) A reconfigurable $S^{3} \mathrm{PR}$ net $\left(N_{4}, M_{40}\right)$.

\section{Deadlock Prevention Policy for Reconfigurable $S^{3}$ PR Net Based on Siphons}

This section presents definitions on siphons in reconfigurable $S^{3} P R$ nets. Next, the siphon control method based on place invariants is introduced. Finally, a deadlock prevention algorithm is proposed to solve the deadlock problems in reconfigurable $S^{3} \mathrm{PR}$ nets.

Definition 29. Let $N_{R}$ be a reconfigurable $S^{3} P R$ net with $N_{R}=\left(\left(N, M_{0}\right), \mathcal{R}\right)$. Let $N_{1}$ be a state of $N_{R}$ with $N_{1}$ $=\left(P_{C 1}, T_{1}, F_{1}, W_{1}, M_{10}, K_{1}\right)$. A place vector of $N_{1}$ is expressed as a column vector $I: P_{C 1} \rightarrow Z$ indexed by $P_{C 1}$, and a transition vector of $N_{1}$ is defined as a column vector $\mathrm{J}: T_{1} \rightarrow \boldsymbol{Z}$ indexed by $T_{1}$, where $\boldsymbol{Z}=\{\ldots,-2,-1,0$, $1,2, \ldots\}$.

Definition 30. Let $N_{R}$ be a reconfigurable $S^{3} P R$ net with $N_{R}=\left(\left(N, M_{0}\right), \mathcal{R}\right)$. Let $N_{1}$ be a state of $N_{R}$ with $N_{1}$ $=\left(P_{C 1}, T_{1}, F_{1}, W_{1}, M_{10}, K_{1}\right)$. A place vector I of $N_{1}$ is expressed as a place invariant (PI) if $I^{T}$. [ $\left.N_{1}\right]=\boldsymbol{0}^{T}$ and I $\neq \mathbf{0}$, and a transition vector of $N_{1}$ is defined as a transition invariant (TI) if $\left[N_{1}\right] . J=\mathbf{0}$ and $J \neq \mathbf{0}$. 
Definition 31. Let $N_{R}$ be a reconfigurable $S^{3} P R$ net with $N_{R}=\left(\left(N, M_{0}\right)\right.$, R). Let $N_{1}$ be a state of $N_{R}$ with $N_{1}$ $=\left(P_{C 1}, T_{1}, F_{1}, W_{1}, M_{10}, K_{1}\right)$. A place invariant $I$ of $N_{1}$ is expressed as a place semi-flow if each element of $I$ is non-negative. $\|I\|=\{p \mid I(p) \neq 0\}$ is said to be the support of place invariant of $I .\|I\|^{+}=\{p \mid I(p)>0\}$ is said to be the positive support of place invariant $I .\|I\|^{-}=\{p \mid I(p)<0\}$ is said to be the negative support of place invariant I. I is a minimal place invariant if $\|I\|$ is not a superset of the support of any other one and its components are mutually prime.

Definition 32. Let $N_{R}$ be a reconfigurable $S^{3} P R$ net with $N_{R}=\left(\left(N, M_{0}\right)\right.$, R). Let $N_{1}$ be a state of $N_{R}$ with $N_{1}=\left(P_{C 1}, T_{1}, F_{1}, W_{1}, M_{10}, K_{1}\right)$. A transition invariant $J$ of $N_{1}$ is expressed as a transition semi-flow if each element of $J$ is non-negative. $\|J\|=\{t \mid J(t) \neq 0\}$ is said to be the support of transition invariant of J. $\|J\|^{+}=\{t \mid J(t)>$ $0\}$ is said to be the positive support of transition invariant $J .\|J\|^{-}=\{t \mid J(t)<0\}$ is said to be the negative support of transition invariant $J$. $J$ is a minimal transition invariant, if $\|J\|$ is not a superset of the support of any other one, and its components are mutually prime.

Definition 33. Let $N_{R}$ be a reconfigurable $S^{3} P R$ net with $N_{R}=\left(\left(N, M_{0}\right), \mathcal{R}\right)$. Let $N_{1}$ be a state of $N_{R}$ with $N_{1}$ $=\left(P_{C 1}, T_{1}, F_{1}, W_{1}, M_{10}, K_{1}\right) . l_{i}$ is said to be the coefficients of place invariant I if for all $p_{i} \in P_{C 1}, l_{i}=I\left(p_{i}\right)$.

Definition 34. Let $N_{R}$ be a reconfigurable $S^{3} P R$ net with $N_{R}=\left(\left(N, M_{0}\right), \mathcal{R}\right)$. Let $N_{1}$ be a state of $N_{R}$ with $N_{1}$ $=\left(P_{C 1}, T_{1}, F_{1}, W_{1}, M_{10}, K_{1}\right)$. A non-empty set $S \subseteq P_{C 1}$ is called a siphon in $N_{1}$ if $\bullet S \subseteq S \bullet S \subseteq P_{C 1}$ is called a trap in $N_{1}$ if $S^{\bullet} \subseteq \bullet$. $S \subseteq P_{C 1}$ is called a minimal siphon (trap) if a siphon (trap) contains no other siphons. A minimal siphon $S$ is called a strict minimal siphon if $S^{\bullet} \subsetneq \bullet$. Let $\Pi=\left\{S_{1}, S_{2}, S_{3}, \ldots, S_{k}\right\}$ be a set of strict minimal siphons of $N_{1}$. We have $S=S_{A} \cup S_{R}, S_{R}=S \cap P_{R}$, and $S_{A}=S \backslash S_{R}$, where $S_{A}$ and $S_{R}$ are sets of operations and resources places, respectively.

Definition 35. Let $N_{R}$ be a reconfigurable $S^{3} P R$ net with $N_{R}=\left(\left(N, M_{0}\right)\right.$, R). Let $N_{1}$ be a state of $N_{R}$ with $N_{1}=$ $\left(P_{C 1}, T_{1}, F_{1}, W_{1}, M_{10}, K_{1}\right)$. A siphon $S$ in $N_{1}$ is called marked at marking $M$ if $\sum_{p \in S} M(p) \geq 1$, and otherwise is called unmarked at marking $M$.

Definition 36. Let $N_{R}$ be a reconfigurable $S^{3} P R$ net with $N_{R}=\left(\left(N, M_{0}\right), \mathcal{R}\right)$. Let $N_{1}$ be a state of $N_{R}$ with $N_{1}$ $=\left(P_{C 1}, T_{1}, F_{1}, W_{1}, M_{10}, K_{1}\right)$. A siphon $S$ in $N_{1}$ is called an emptiable siphon if there exists $M \in R\left(N_{1}, M_{10}\right)$ such that $\sum_{p \in S} M(p)=0$, and otherwise is called non-emptiable siphon.

Theorem 4. Let $N_{R}$ be a reconfigurable $S^{3} P R$ net with $N_{R}=\left(\left(N, M_{0}\right), \mathcal{R}\right)$. Let $N_{1}$ be a state of $N_{R}$ with $N_{1}=$ $\left(P_{C 1}, T_{1}, F_{1}, W_{1}, M_{10}, K_{1}\right)$ and $\Pi$ the set of $N_{1}$ siphons. The net $N_{1}$ is deadlock-free if for all $S \in \Pi$, for all $M \in$ $R\left(N_{1}, M_{10}\right), \sum_{p \in S} M(p) \geq 1$.

Proof. Let $S$ be a siphon in $N_{1}$ and $p \in S$. $p$ is marked at marking $M$ and satisfies $\sum_{p \in S} M(p) \geq 1$. The net $N_{1}$ has at least one transition $t$ enabled at any marking reachable from $M$ and $S$ is never be an unmarked, and it is therefore deadlock-free.

Theorem 5. Let $N_{R}$ be a reconfigurable $S^{3} P R$ net with $N_{R}=\left(\left(N, M_{0}\right), \mathcal{R}\right)$. Let $N_{1}$ be a state of $N_{R}$ with $N_{1}=$ $\left(P_{C 1}, T_{1}, F_{1}, W_{1}, M_{10}, K_{1}\right)$ and $\Pi$ the set of $N_{1}$ siphons. The net $\left(N_{1}, M_{10}\right)$ is in a deadlock state, i.e., $M$ is a dead marking of $N_{1}$. Then, $\left\{p \in P_{C 1} \mid M(p)=0\right\}$ is a siphon $S$.

Proof. Since $M$ is a dead marking, each $t$ has an empty input place $p$ at $M, \forall p \in \bullet^{\bullet}, M(p)<W(p, t)$, and thus $S^{\bullet}$ includes each transition of $N_{1}$. In fact, we have ${ }^{\bullet} S \subseteq S^{\bullet}$. Therefore, $S$ is a siphon. Since the net has at least one transition $t \in T_{1}, S$ is not an empty set.

Corollary 3. Let $N_{R}$ be a reconfigurable $S^{3} P R$ net with $N_{R}=\left(\left(N, M_{0}\right), \mathcal{R}\right)$. Let $N_{1}$ be a state of $N_{R}$ with $N_{1}=$ $\left(P_{C 1}, T_{1}, F_{1}, W_{1}, M_{10}, K_{1}\right)$, a deadlocked $N_{1}$ net includes at least one unmarked siphon $S$. 
Corollary 4. Let $N_{R}$ be a reconfigurable $S^{3} P R$ net with $N_{R}=\left(\left(N, M_{0}\right), \mathcal{R}\right)$. Let $N_{1}$ be a state of $N_{R}$ with $N_{1}=$ $\left(P_{C 1}, T_{1}, F_{1}, W_{1}, M_{10}, K_{1}\right), N_{1}$ is a deadlocked net at marking $M$. Then, $N_{1}$ has at least one unmarked siphon $S$ such that for all $p \in S$, there exists $t \in p^{\bullet}$ such that $W_{1}(p, t)>M(p)$.

To develop a deadlock prevention policy for reconfigurable $S^{3} \mathrm{PR}$ net, we reviewed the approach of designing a control place (monitor) for a place invariant developed by Yamalidou et al. [40]. Then we develop a deadlock prevention policy for reconfigurable $S^{3} \mathrm{PR}$ net to achieve an optimal place invariant. Yamalidou et al. propose a computationally efficient method based on place invariants that enforces algebraic constraints on the elements of a marking of a net system by constructing control places. The control purpose is to ensure a siphon to be a marked siphon, i.e., ensure a siphon be non-emptiable at all elements of a marking.

Assume that a reconfigurable $S^{3} P R$ net with $N_{R}=\left(\left(N, M_{o}\right), \mathcal{R}\right)$ and $N_{k}$ (state of $\left.N_{R}\right)$ with $N_{k}=$ $\left(P_{C k}, T_{k}, F_{k}, W_{k}, M_{k o}, K_{k}\right), k=1,2, \ldots,|\mathcal{R}|$ is a net to be controlled, which includes $n$ places and $m$ transitions. Let $\left[N_{k}\right]$ be the incidence matrix of a plant reconfigurable $S^{3} P R$ net. The control places can be represented by $\left[N_{c}\right]$ a matrix that shows the connection relationship between control places to transitions of the net $N_{k}$. The controlled net with incidence matrix $[N]$ comprises both the original reconfigurable $S^{3} \mathrm{PR}$ net and the monitors, i.e.,

$$
[N]=\left[\begin{array}{c}
N_{k} \\
N_{c}
\end{array}\right]
$$

The control purpose is to impose a set of linear constraints to prevent unwanted markings being reached. The constraints are formulated in a matrix form:

$$
\mathcal{L} \cdot M \geq \mathcal{B}
$$

where $M$ denotes the marking vector of net $N_{k}, \mathcal{L}$ is an integer $n_{c} \times n$ matrix $\left(n_{c}\right.$ - the number of constraints), and $\mathcal{B}$ is an integer column vector. After the introduction of a non-negative slack variable that corresponds to the initial marking $M_{k o}$ of $N_{k}$, constraint (5) can be reformulated as:

$$
M_{c o}=\mathcal{B}-\mathcal{L} \cdot M_{k o} .
$$

where $M_{c o}$ represents the initial marking of monitor $c$.

If $\left[N_{k}\right]$ is the incidence matrix, we have: $M_{k}=M_{k o}+\left[N_{k}\right] . \vec{\delta}$. Therefore, $M_{c}=\mathcal{B}-\mathcal{L} \cdot\left(M_{k o}+\left[N_{k}\right] . \vec{\delta}\right)$, which also can be reformulated as:

$$
M_{c}=M_{c o}+\left(-\mathcal{L} \cdot\left[N_{k}\right] \cdot \vec{\delta}\right)
$$

The place invariant computed by (5) must meet the place invariant equation $I^{\mathrm{T}}[N]=\mathbf{0}^{\mathrm{T}}$. Therefore, the monitor $\left[N_{c}\right]$ can be formulated as:

$$
\left[N_{c}\right]=-\mathcal{L} \cdot\left[N_{k}\right]
$$

Consequently, $M_{c}$ may be considered as a marking of some additional monitors, where the supervised reconfigurable $S^{3} \mathrm{PR}$ net has an incidence matrix $[N]=\left[\begin{array}{c}N_{k} \\ N_{c}\end{array}\right]$, and a marking vector $M=\left[\begin{array}{l}M_{k} \\ M_{c}\end{array}\right]$. 
Theorem 6. Let $N_{R}$ be a reconfigurable $S^{3} P R$ net with $N_{R}=\left(\left(N, M_{0}\right), \mathcal{R}\right)$. Let $N_{k}$ be a state of $N_{R}$ with $N_{k}=\left(P_{C k}, T_{k}, F_{k}, W_{k}, M_{k o}, K_{k}\right)$, incidence matrix $\left[N_{k}\right]$ and initial marking $M_{k o}$ be given. A set of $n_{c}$ linear constraints $\mathcal{L} \cdot M_{k} \geq \mathcal{B}$ are to be imposed. If $\mathcal{B}-\mathcal{L} \cdot M_{k} \geq 0$ then a Petri net controller with incidence matrix $\left[N_{c}\right]=-L \cdot\left[N_{k}\right]$ and initial marking $M_{c o}=\mathcal{B}-\mathcal{L} \cdot M_{k o}$ enforces the constraint $\mathcal{L} \cdot M_{k} \geq \mathcal{B}$ when included in the closed loop system $[N]=\left[\begin{array}{l}N_{k} \\ N_{c}\end{array}\right]$. In addition, the controller is maximally permissive.

Proof. See $[40,41]$.

Now, we consider the place invariant approach to control the siphon. Let $S$ be an unmarked siphon. The control purpose is to ensure that $S$ is never unmarked through the system evolution $(N$, $M_{o}$ ) and eliminate markings that break the linear constraint (5) from the reachable markings.

Let $V_{S} \backslash S \in \Pi$ be the monitor resulting from controlling the siphon $S$. There are siphons $S$ such that if $\sum_{p \in S} M_{o}(p) \geq 1$ for the initial marking $M_{o}$, then $\sum_{p \in S} M(p) \geq 1$ for all reachable markings $M$. Therefore, a siphon $S$ does not require control. In order to reduce the supervisor's complexity, these siphons are identified and no monitors are added. Thus, we have two sets of constraints: $\mathcal{L} . M \geq \mathcal{B}$ and $\mathcal{L}_{0} . M \geq \mathcal{B}_{0}$ rather than a single set of constraints $\mathcal{L} . M \geq \mathcal{B}$. The deadlock prevention supervision of the original net needs enforcing $\mathcal{L} . M \geq \mathcal{B}$ and selecting an initial marking $M_{o}$ such that $\mathcal{L}_{0} \cdot M_{o} \geq \mathcal{B}_{o}$ and $\mathcal{L} . M_{o} \geq \mathcal{B}$. The constraints $\mathcal{L}_{0} \cdot M \geq \mathcal{B}_{0}$ are the constraints that all reachable markings satisfy when the initial markings satisfy them. Therefore, there are two cases to control a siphon:

If $V_{S} \bullet \subseteq{ }^{\bullet}$, then $S$ does not require monitor and $V_{S}$ is not assigned to a net $N$. Furthermore, $V_{S} \subseteq$ - $S$ if and only if $S$ is a trap. Thus, when $S$ is also a siphon, it is (trap) controlled for all initial markings $M_{o}$ that satisfy $\sum_{p \in S} M_{o}(p) \geq 1$. Therefore, a siphon $S$ is assigned to $\left(\mathcal{L}_{o} ; \mathcal{B}_{0}\right)$.

A. If $V_{S} \bullet{ }^{\bullet} S$, then $S$ needs a monitor and $V_{S}$ is assigned to $N$. Therefore, the $S$ is assigned to $(\mathcal{L} ; \mathcal{B})$.

Definition 37. Let $N_{R}$ be a reconfigurable $S^{3} P R$ net with $N_{R}=\left(\left(N, M_{o}\right), \mathcal{R}\right)$. Let $N_{k}$ be a state in $N_{R}$ with $N_{k}$ $=\left(P_{C k}, T_{k}, F_{k}, W_{k}, M_{k o}, K_{k}\right)$. A siphon $S$ in $N_{k}$ is called controlled if for all $M \in R\left(N_{k}, M_{k o}\right), \sum_{p \in S} M(p) \geq 1$ and satisfy $\mathcal{L} . M \geq \mathcal{B}$ and $\mathcal{L}_{0} . M \geq \mathcal{B}_{0}$.

Definition 38. Let $N_{R}$ be a reconfigurable $S^{3} P R$ net with $N_{R}=\left(\left(N, M_{0}\right)\right.$, R). Let $N_{k}$ be a state in $N_{R}$ with $N_{k}$ $=\left(P_{C k}, T_{k}, F_{k}, W_{k}, M_{k o}, K_{k}\right)$. The deadlock controller for $\left(N_{k}, M_{o k}\right)$ is expressed as $\left(V, M_{V o}\right)=\left(P_{V}, T_{V}, F_{V}\right.$, $\left.M_{V o}\right)$, where (1) $P_{V}=\left\{V_{S} \backslash S \in \Pi\right\}$ is set of monitors. (2) $T_{V}=\left\{t \backslash t \in V_{S} \cup V_{S} \bullet\right\}$. (3) $F_{V} \subseteq\left(P_{V} \times T_{V}\right) \cup\left(T_{V} \times\right.$ $\left.P_{V}\right)$ is called a flow relation of $V$. (4) for all $V_{S} \in P_{V}, M_{V o}\left(V_{S}\right)=\mathcal{B}-\mathcal{L} \cdot M_{k o}\left(V_{s}\right)$, where $M_{V o}\left(V_{S}\right)$ is called an initial marking of a monitor. $\left(N_{R C}, M_{R C_{0}}\right)$ is said to be a controlled reconfigurable $S^{3} P R$ net resulting from the integration of $\left(N_{k}, M_{k o}\right)$ and $\left(V, M_{V o}\right)$, expressed as $\left(N_{k}, M_{k o}\right) \|\left(V, M_{V o}\right)$, where $N_{R C}=\left(P_{R C}, T_{R C}, F_{R C}, W_{R C}\right.$, $\left.M_{R C o}, K_{R C}\right), P_{R C}=P_{C k} \cup P_{V}, T_{R C}=T_{k} \cup T_{V}, F_{R C}:\left(P_{R C} \times T_{R C}\right) \cup\left(T_{R C} \times P_{R C}\right) \rightarrow I N$ is called flow relations, $W_{R C}:\left(P_{R C} \times T_{R C}\right) \cup\left(T_{R C} \times P_{R C}\right) \rightarrow I N$ is a mapping that assigns a weight to an arc, $M_{R C o}: P_{R C} \rightarrow I N$ is the initial marking, and $K_{R C}: P_{R C} \rightarrow I N$ is the function of capacity that assigns to each place $p$ the maximal number of tokens $K_{R C}(p)$.

Based on the concept of place invariant and siphon control, the deadlock prevention algorithm for reconfigurable $\mathrm{S}^{3} \mathrm{PR}$ net is developed as follows: 


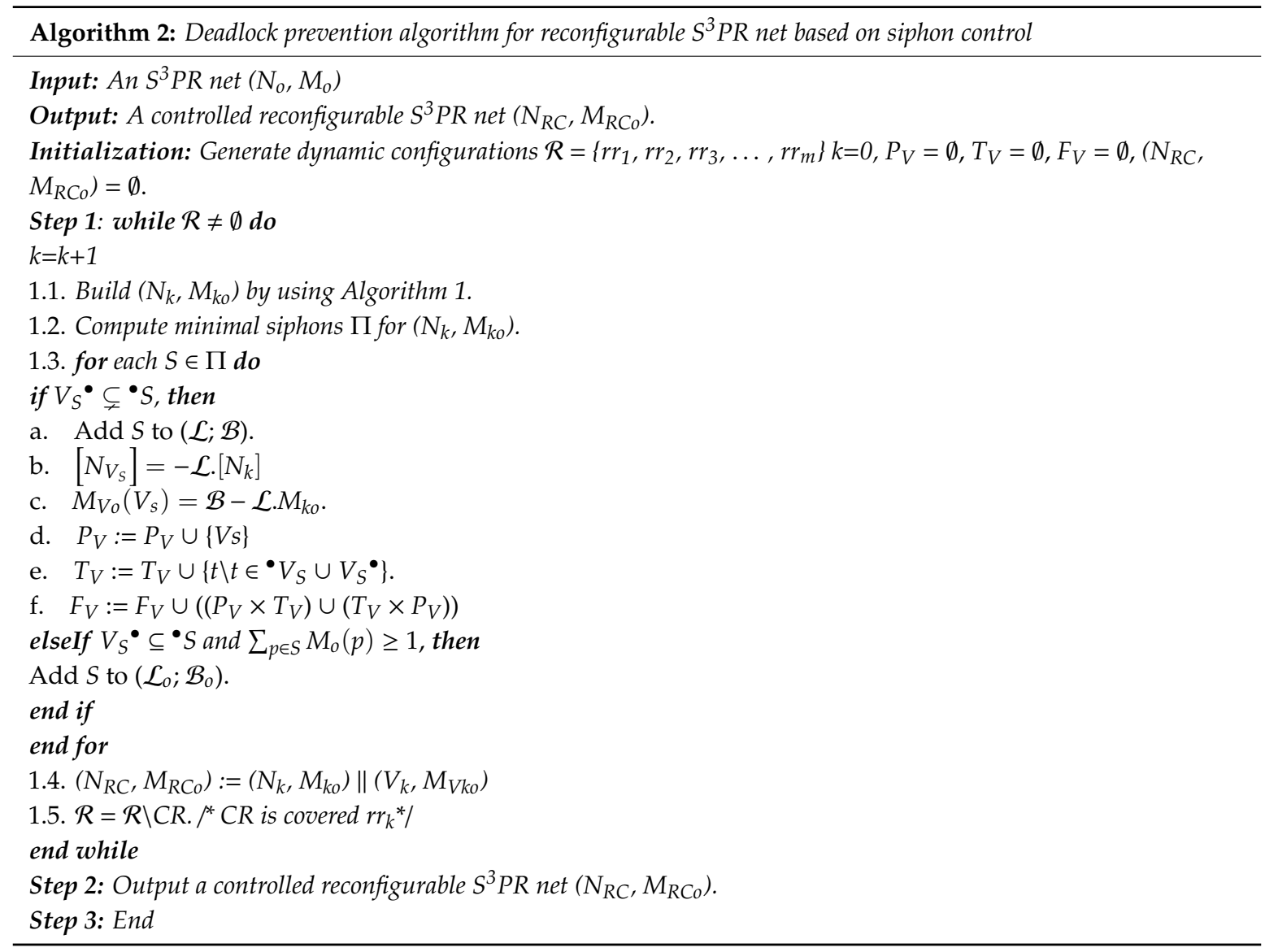

To illustrate the proposed Algorithm 2, reconsider the initial $S^{3} \mathrm{PR}$ net $\left(N_{0}, M_{0}\right)$ illustrated in Figure 2. The initial net has four minimal siphons $S_{1}=\left\{p_{1}, p_{2}, p_{3}, p_{4}\right\}, S_{2}=\left\{p_{3}, p_{5}\right\}, S_{3}=\left\{p_{2}, p_{4}, p_{6}\right\}$, and $S_{4}=\left\{p_{4}, p_{5}, p_{6}\right\}$. The $N_{o}$ incidence matrix is

$$
\left[N_{o}\right]=\left[\begin{array}{cccc}
-1 & 0 & 0 & 1 \\
1 & -1 & 0 & 0 \\
0 & 1 & -1 & 0 \\
0 & 0 & 1 & -1 \\
0 & -1 & 1 & 0 \\
-1 & 1 & -1 & 1
\end{array}\right]
$$

while its initial marking is:

$$
M_{0}=\left[\begin{array}{llllll}
5 & 0 & 0 & 0 & 1 & 1
\end{array}\right]^{\mathrm{T}}
$$

$S_{4}$ creates monitor $V_{S 1}$, therefore one monitor $V_{S 1}$ is added, which enforces:

$$
M\left(p_{4}\right)+M\left(p_{5}\right)+M\left(p_{6}\right) \geq 1
$$

The following place invariant is generated:

$$
M\left(V_{S 1}\right)=M\left(p_{4}\right)+M\left(p_{5}\right)+M\left(p_{6}\right)-1
$$

The current matrices $\mathcal{L}$ and $\mathcal{B}$ represent the Equation (12).

$$
\mathcal{L}=\left[\begin{array}{llllll}
0 & 0 & 0 & 1 & 1 & 1
\end{array}\right], \mathcal{B}=[1]
$$


while the others minimal siphons create constraints in $\left(\mathcal{L}_{0} ; \mathcal{B}_{0}\right)$.

$$
\mathcal{L}_{o}=\left[\begin{array}{llllll}
1 & 1 & 1 & 1 & 0 & 0 \\
0 & 0 & 1 & 0 & 1 & 0 \\
0 & 1 & 0 & 0 & 0 & 1
\end{array}\right], \mathcal{B}_{o}=\left[\begin{array}{l}
1 \\
1 \\
1
\end{array}\right]
$$

The controller net incidence matrix is calculated by Equation (8):

$$
\left[N_{V_{S}}\right]=-\mathcal{L} \cdot\left[N_{o}\right]=\left[\begin{array}{llll}
-1 & 0 & 1 & 0
\end{array}\right]
$$

The controller's initial place marking is calculated as:

$$
M_{o}\left(V_{S 1}\right)=M_{o}\left(p_{4}\right)+M_{o}\left(p_{5}\right)+M_{o}\left(p_{6}\right)-1=1
$$

The controlled net of $\left(N_{o}, M_{o}\right)$ is illustrated in Figure 7. The place and arcs of the controller are shown with blue lines.

Now, reconsider the reconfigured $S^{3} P R$ net by addition of new machine $\left(N_{1}, M_{10}\right)$ illustrated in Figure 3c. The reconfigured net has seven minimal siphons $S_{1}=\left\{p_{3}, p_{5}\right\}, S_{2}=\left\{p_{7}, p_{9}\right\}, S_{3}=\left\{p_{2}, p_{4}, p_{6}\right.$, $\left.p_{8}\right\}, S_{4}=\left\{p_{4}, p_{5}, p_{6}, p_{8}\right\}, S_{5}=\left\{p_{2}, p_{6}, p_{8}, p_{9}\right\}, S_{6}=\left\{p_{5}, p_{6}, p_{8}, p_{9}\right\}$, and $S_{7}=\left\{p_{1}, p_{2}, p_{3}, p_{4}, p_{7}, p_{8}\right\}$.

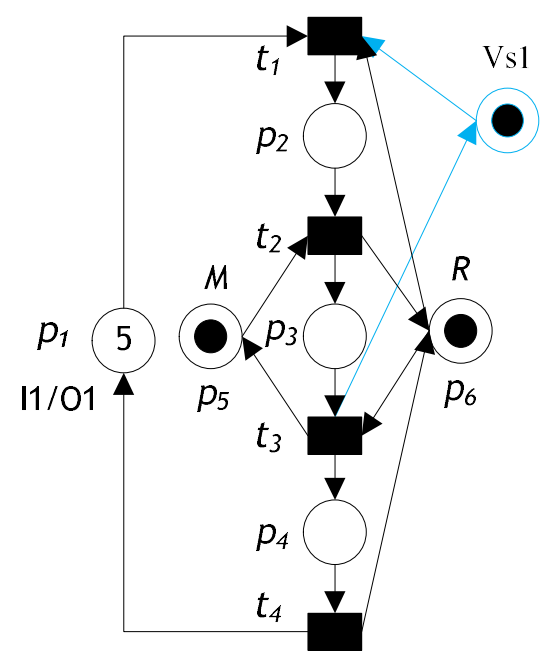

Figure 7. Controlled $S^{3} \mathrm{PR}$ net by Algorithm 2.

The $N_{1}$ incidence matrix is:

$$
\left[N_{1}\right]=\left[\begin{array}{cccccc}
-1 & 0 & 0 & 0 & 0 & 1 \\
1 & -1 & 0 & 0 & 0 & 0 \\
0 & 1 & -1 & 0 & 0 & 0 \\
0 & 0 & 1 & -1 & 0 & 0 \\
0 & -1 & 1 & 0 & 0 & 0 \\
-1 & 1 & -1 & 1 & -1 & 1 \\
0 & 0 & 0 & 1 & -1 & 0 \\
0 & 0 & 0 & 0 & 1 & -1 \\
0 & 0 & 0 & -1 & 1 & 0
\end{array}\right]
$$

while its initial marking is:

$$
M_{1 O}=\left[\begin{array}{lllllllll}
5 & 0 & 0 & 0 & 1 & 1 & 0 & 0 & 1
\end{array}\right]^{\mathrm{T}}
$$


$S_{4}, S_{5}$, and $S_{6}$ create monitor $V_{S 1}, V_{S 2}$, and $V_{S 3}$, respectively. Thus, three monitors are added, $V_{S 1}$, $V_{S 2}$, and $V_{S 3}$, which enforce:

$$
\begin{aligned}
& M\left(p_{4}\right)+M\left(p_{5}\right)+M\left(p_{6}\right)+M\left(p_{8}\right) \geq 1 \\
& M\left(p_{2}\right)+M\left(p_{6}\right)+M\left(p_{8}\right)+M\left(p_{9}\right) \geq 1 \\
& M\left(p_{5}\right)+M\left(p_{6}\right)+M\left(p_{8}\right)+M\left(p_{9}\right) \geq 1
\end{aligned}
$$

The following place invariants are accordingly generated:

$$
\begin{aligned}
& M\left(V_{S 1}\right)=M\left(p_{4}\right)+M\left(p_{5}\right)+M\left(p_{6}\right)+M\left(p_{8}\right)-1 \\
& M\left(V_{S 2}\right)=M\left(p_{2}\right)+M\left(p_{6}\right)+M\left(p_{8}\right)+M\left(p_{9}\right)-1 \\
& M\left(V_{S 3}\right)=M\left(p_{5}\right)+M\left(p_{6}\right)+M\left(p_{8}\right)+M\left(p_{9}\right)-1
\end{aligned}
$$

The current matrices $\mathcal{L}$ and $\mathcal{B}$ represent the Equations (18)-(20).

$$
\mathcal{L}=\left[\begin{array}{lllllllll}
0 & 0 & 0 & 1 & 1 & 1 & 0 & 1 & 0 \\
0 & 1 & 0 & 0 & 0 & 1 & 0 & 1 & 1 \\
0 & 0 & 0 & 0 & 1 & 1 & 0 & 1 & 1
\end{array}\right], \mathcal{B}=\left[\begin{array}{l}
1 \\
1 \\
1
\end{array}\right]
$$

while the other minimal siphons create constraints in $\left(\mathcal{L}_{0} ; \mathcal{B}_{0}\right)$.

$$
\mathcal{L}_{o}=\left[\begin{array}{lllllllll}
0 & 0 & 1 & 0 & 1 & 0 & 0 & 0 & 0 \\
0 & 0 & 0 & 0 & 0 & 0 & 1 & 0 & 1 \\
0 & 1 & 0 & 1 & 0 & 1 & 0 & 1 & 0 \\
1 & 1 & 1 & 1 & 0 & 0 & 1 & 1 & 0
\end{array}\right], \mathcal{B}_{0}=\left[\begin{array}{l}
1 \\
1 \\
1 \\
1
\end{array}\right]
$$

The controller's net incidence matrix is calculated by Equation (12);

$$
\left[N_{V_{S}}\right]=-\mathcal{L} \cdot\left[N_{1}\right]=\left[\begin{array}{cccccc}
-1 & 0 & 1 & 0 & 0 & 0 \\
0 & 0 & -1 & 0 & 1 & 0 \\
-1 & 0 & 0 & 0 & 1 & 0
\end{array}\right]
$$

The initial marking controllers are calculated as:

$$
\begin{aligned}
& M_{o}\left(V_{S 1}\right)=M_{o}\left(p_{4}\right)+M_{o}\left(p_{5}\right)+M_{o}\left(p_{6}\right)+M_{o}\left(p_{8}\right)-1=1 \\
& M_{o}\left(V_{S 2}\right)=M_{o}\left(p_{2}\right)+M_{o}\left(p_{6}\right)+M_{o}\left(p_{8}\right)+M_{o}\left(p_{9}\right)-1=1 \\
& M_{o}\left(V_{S 3}\right)=M_{o}\left(p_{5}\right)+M_{o}\left(p_{6}\right)+M_{o}\left(p_{8}\right)+M_{o}\left(p_{9}\right)-1=2
\end{aligned}
$$

The controlled reconfigurable net of $\left(N_{1}, M_{10}\right)$ is illustrated in Figure 8. The place and arcs of the controllers are shown with blue lines. 


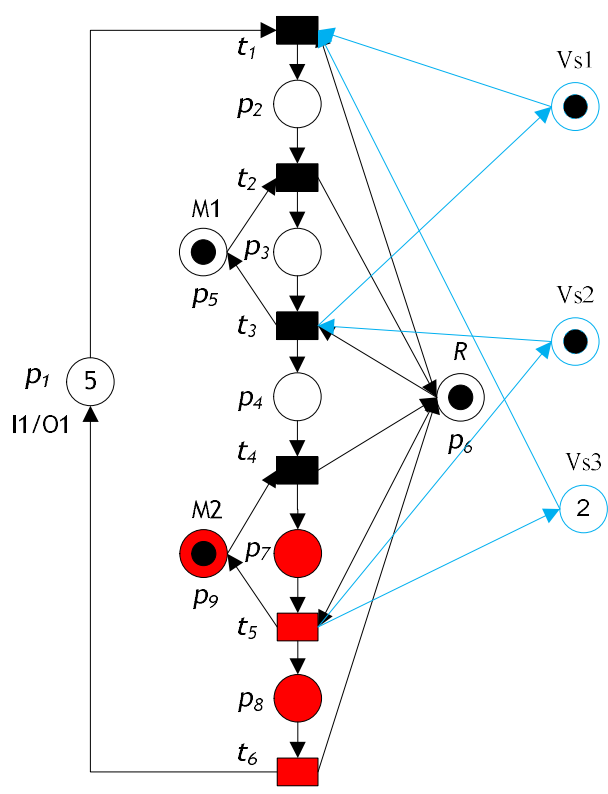

Figure 8. Controlled reconfigurable $S^{3} \mathrm{PR}$ net by addition of new machine.

Then, reconsider the reconfigured $S^{3} P R$ net by addition of new product $\left(N_{2}, M_{20}\right)$ illustrated in Figure 4c. The reconfigured net has 11 minimal siphons $S_{1}=\left\{p_{7}, p_{9}\right\}, S_{2}=\left\{p_{3}, p_{5}, p_{11}\right\}, S_{3}=\left\{p_{10}, p_{11}\right.$, $\left.p_{12}, p_{13}\right\}, S_{4}=\left\{p_{4}, p_{5}, p_{6}, p_{8}, p_{12}\right\}, S_{5}=\left\{p_{5}, p_{6}, p_{8}, p_{9}, p_{12}\right\}, S_{6}=\left\{p_{1}, p_{2}, p_{3}, p_{4}, p_{7}, p_{8}\right\}, S_{7}=\left\{p_{2}, p_{4}, p_{6}, p_{8}\right.$, $\left.p_{10}, p_{12}\right\}, S_{8}=\left\{p_{2}, p_{6}, p_{8}, p_{9}, p_{10}, p_{12}\right\}, S_{9}=\left\{p_{4}, p_{5}, p_{6}, p_{8}, p_{12}\right\}, S_{10}=\left\{p_{5}, p_{6}, p_{8}, p_{9}, p_{12}\right\}$, and $S_{11}=\left\{p_{2}, p_{6}\right.$, $\left.p_{8}, p_{9}, p_{10}, p_{12}\right\}$. The $N_{2}$ incidence matrix is

$$
\left[N_{2}\right]=\left[\begin{array}{cccccccccc}
-1 & 0 & 0 & 0 & 0 & 1 & 0 & 0 & 0 & 0 \\
1 & -1 & 0 & 0 & 0 & 0 & 0 & 0 & 0 & 0 \\
0 & 1 & -1 & 0 & 0 & 0 & 0 & 0 & 0 & 0 \\
0 & 0 & 1 & -1 & 0 & 0 & 0 & 0 & 0 & 0 \\
0 & -1 & 1 & 0 & 0 & 0 & 0 & -1 & 1 & 0 \\
-1 & 1 & -1 & 1 & -1 & 1 & -1 & 1 & -1 & 1 \\
0 & 0 & 0 & 1 & -1 & 0 & 0 & 0 & 0 & 0 \\
0 & 0 & 0 & 0 & 1 & -1 & 0 & 0 & 0 & 0 \\
0 & 0 & 0 & -1 & 1 & 0 & 0 & 0 & 0 & 0 \\
0 & 0 & 0 & 0 & 0 & 0 & 1 & -1 & 0 & 0 \\
0 & 0 & 0 & 0 & 0 & 0 & 0 & 1 & -1 & 0 \\
0 & 0 & 0 & 0 & 0 & 0 & 0 & 0 & 1 & -1 \\
0 & 0 & 0 & 0 & 0 & 0 & -1 & 0 & 0 & 1
\end{array}\right]
$$

while its initial marking is:

$$
M_{2 O}=\left[\begin{array}{lllllllllllll}
5 & 0 & 0 & 0 & 1 & 1 & 0 & 0 & 1 & 0 & 0 & 0 & 5
\end{array}\right]^{\mathrm{T}}
$$

$S_{4}, S_{5}$, and $S_{8}$ create monitors $V_{S 1}, V_{S 2}$, and $V_{S 3}$, respectively. Thus, three monitors are added, $V_{S 1}, V_{S 2}$, and $V_{S 3}$, which enforce:

$$
\begin{gathered}
M\left(p_{4}\right)+M\left(p_{5}\right)+M\left(p_{6}\right)+M\left(p_{8}\right)+M\left(p_{12}\right) \geq 1 \\
M\left(p_{5}\right)+M\left(p_{6}\right)+M\left(p_{8}\right)+M\left(p_{9}\right)+M\left(p_{12}\right) \geq 1 \\
M\left(p_{2}\right)+M\left(p_{6}\right)+M\left(p_{8}\right)+M\left(p_{9}\right)+M\left(p_{10}\right)+M\left(p_{12}\right) \geq 1
\end{gathered}
$$


The following place invariants are accordingly generated:

$$
\begin{gathered}
M\left(V_{S 1}\right)=M\left(p_{4}\right)+M\left(p_{5}\right)+M\left(p_{6}\right)+M\left(p_{8}\right)+M\left(p_{12}\right)-1 \\
M\left(V_{S 2}\right)=M\left(p_{5}\right)+M\left(p_{6}\right)+M\left(p_{8}\right)+M\left(p_{9}\right)+M\left(p_{12}\right)-1 \\
M\left(V_{S 3}\right)=M\left(p_{2}\right)+M\left(p_{6}\right)+M\left(p_{8}\right)+M\left(p_{9}\right)+M\left(p_{10}\right)+M\left(p_{12}\right)-1
\end{gathered}
$$

The current matrices $\mathcal{L}$ and $\mathcal{B}$ represent the Equations (29)-(31).

$$
\mathcal{L}=\left[\begin{array}{lllllllllllll}
0 & 0 & 0 & 1 & 1 & 1 & 0 & 1 & 0 & 0 & 0 & 1 & 0 \\
0 & 0 & 0 & 0 & 1 & 1 & 0 & 1 & 1 & 0 & 0 & 1 & 0 \\
0 & 1 & 0 & 0 & 0 & 1 & 0 & 1 & 1 & 1 & 0 & 1 & 0
\end{array}\right], \mathcal{B}=\left[\begin{array}{l}
1 \\
1 \\
1
\end{array}\right]
$$

while the other minimal siphons create constraints in $\left(\mathcal{L}_{0} ; \mathcal{B}_{0}\right)$.

$$
\mathcal{L}_{o}=\left[\begin{array}{lllllllllllll}
0 & 0 & 0 & 0 & 0 & 0 & 1 & 0 & 1 & 0 & 0 & 0 & 0 \\
0 & 0 & 1 & 0 & 1 & 0 & 0 & 0 & 0 & 0 & 1 & 0 & 0 \\
0 & 0 & 0 & 0 & 0 & 0 & 0 & 0 & 0 & 1 & 1 & 1 & 1 \\
1 & 1 & 1 & 1 & 0 & 0 & 1 & 1 & 0 & 0 & 0 & 0 & 0 \\
0 & 1 & 0 & 1 & 0 & 1 & 0 & 1 & 0 & 1 & 0 & 1 & 0 \\
0 & 0 & 0 & 1 & 1 & 1 & 0 & 1 & 0 & 0 & 0 & 1 & 0 \\
0 & 0 & 0 & 0 & 1 & 1 & 0 & 1 & 1 & 0 & 0 & 1 & 0 \\
0 & 1 & 0 & 0 & 0 & 1 & 0 & 1 & 1 & 1 & 0 & 1 & 0
\end{array}\right], \mathcal{B}_{o}=\left[\begin{array}{l}
1 \\
1 \\
1 \\
1 \\
1 \\
1 \\
1 \\
1
\end{array}\right]
$$

The controller's net incidence matrix is calculated by Equation (12);

$$
\left[N_{V_{S}}\right]=-\mathcal{L} \cdot\left[N_{20}\right]=\left[\begin{array}{cccccccccc}
-1 & 0 & 1 & 0 & 0 & 0 & -1 & 0 & 1 & 0 \\
-1 & 0 & 0 & 0 & 1 & 0 & -1 & 0 & 1 & 0 \\
0 & 0 & -1 & 0 & 1 & 0 & 0 & 0 & 0 & 0
\end{array}\right]
$$

The initial marking controllers are calculated as:

$$
\begin{gathered}
M_{\mathrm{o}}\left(V_{S 1}\right)=M_{\mathrm{o}}\left(p_{4}\right)+M_{\mathrm{o}}\left(p_{5}\right)+M_{\mathrm{o}}\left(p_{6}\right)+M_{\mathrm{o}}\left(p_{8}\right)+M_{\mathrm{o}}\left(p_{12}\right)-1=1 \\
M_{\mathrm{o}}\left(V_{S 2}\right)=M_{\mathrm{o}}\left(p_{5}\right)+M_{\mathrm{o}}\left(p_{6}\right)+M_{\mathrm{o}}\left(p_{8}\right)+M_{\mathrm{o}}\left(p_{9}\right)+M_{\mathrm{o}}\left(p_{12}\right)-1=2 \\
M_{\mathrm{o}}\left(V_{S 3}\right)=M_{\mathrm{o}}\left(p_{2}\right)+M_{\mathrm{o}}\left(p_{6}\right)+M_{\mathrm{o}}\left(p_{8}\right)+M_{\mathrm{o}}\left(p_{9}\right)+M_{\mathrm{o}}\left(p_{10}\right)+M_{\mathrm{o}}\left(p_{12}\right)-1=1
\end{gathered}
$$

The controlled reconfigurable net of $\left(N_{2}, M_{20}\right)$ is illustrated in Figure 9. The place and arcs of the controllers are shown with blue lines.

Then, reconsider the reconfigured $S^{3} P R$ net by rework $\left(N_{3}, M_{30}\right)$ illustrated in Figure $5 c$. The reconfigured net has 13 minimal siphons $S_{1}=\left\{p_{4}, p_{5}, p_{6}, p_{8}, p_{12}, p_{16}\right\}, S_{2}=\left\{p_{5}, p_{6}, p_{8}, p_{9}\right.$, $\left.p_{12}, p_{16}\right\}, S_{3}=\left\{p_{4}, p_{5}, p_{6}, p_{8}, p_{16}, p_{17}\right\}, S_{4}=\left\{p_{5}, p_{6}, p_{8}, p_{9}, p_{16}, p_{17}\right\}, S_{5}=\left\{p_{2}, p_{6}, p_{8}, p_{9}, p_{10}, p_{12}, p_{15}, p_{16}\right\}$, $S_{6}=\left\{p_{2}, p_{4}, p_{6}, p_{8} p_{10}, p_{15}, p_{16}, p_{17}\right\}, S_{7}=\left\{p_{2}, p_{6}, p_{8}, p_{9}, p_{10}, p_{15}, p_{16}, p_{17}\right\}, S_{8}=\left\{p_{2}, p_{4}, p_{6}, p_{8}, p_{10}, p_{12}\right.$, $\left.p_{15}, p_{16}\right\}, S_{9}=\left\{p_{7}, p_{9}\right\}, S_{10}=\left\{p_{1}, p_{2}, p_{3}, p_{4}, p_{7}, p_{8}\right\}, S_{11}=\left\{p_{3}, p_{5}, p_{11}\right\}, S_{12}=\left\{p_{14}, p_{17}\right\}$, and $S_{13}=\left\{p_{10}, p_{11}\right.$, $\left.p_{12}, p_{13}, p_{14}, p_{15}, p_{16}\right\}$. Siphons $S_{1}-S_{7}$, create monitors $V_{S 1}-V_{S 7}$, respectively. Thus, seven monitors are added, $V_{S 1}-V_{S 7}$, which enforce:

$$
\begin{aligned}
& M\left(p_{4}\right)+M\left(p_{5}\right)+M\left(p_{6}\right)+M\left(p_{8}\right)+M\left(p_{12}\right)+M\left(p_{16}\right) \geq 1 \\
& M\left(p_{5}\right)+M\left(p_{6}\right)+M\left(p_{8}\right)+M\left(p_{9}\right)+M\left(p_{12}\right)+M\left(p_{16}\right) \geq 1 \\
& M\left(p_{4}\right)+M\left(p_{5}\right)+M\left(p_{6}\right)+M\left(p_{8}\right)+M\left(p_{16}\right)+M\left(p_{17}\right) \geq 1 \\
& M\left(p_{5}\right)+M\left(p_{6}\right)+M\left(p_{8}\right)+M\left(p_{9}\right)+M\left(p_{16}\right)+M\left(p_{17}\right) \geq 1
\end{aligned}
$$




$$
\begin{aligned}
& M\left(p_{2}\right)+M\left(p_{6}\right)+M\left(p_{8}\right)+M\left(p_{9}\right)+M\left(p_{10}\right)+M\left(p_{12}\right)+M\left(p_{15}\right)+M\left(p_{16}\right) \geq 1 \\
& M\left(p_{2}\right)+M\left(p_{4}\right)+M\left(p_{6}\right)+M\left(p_{8}\right)+M\left(p_{10}\right)+M\left(p_{15}\right)+M\left(p_{16}\right)+M\left(p_{17}\right) \geq 1 \\
& M\left(p_{2}\right)+M\left(p_{6}\right)+M\left(p_{8}\right)+M\left(p_{9}\right)+M\left(p_{10}\right)+M\left(p_{15}\right)+M\left(p_{16}\right)+M\left(p_{17}\right) \geq 1
\end{aligned}
$$

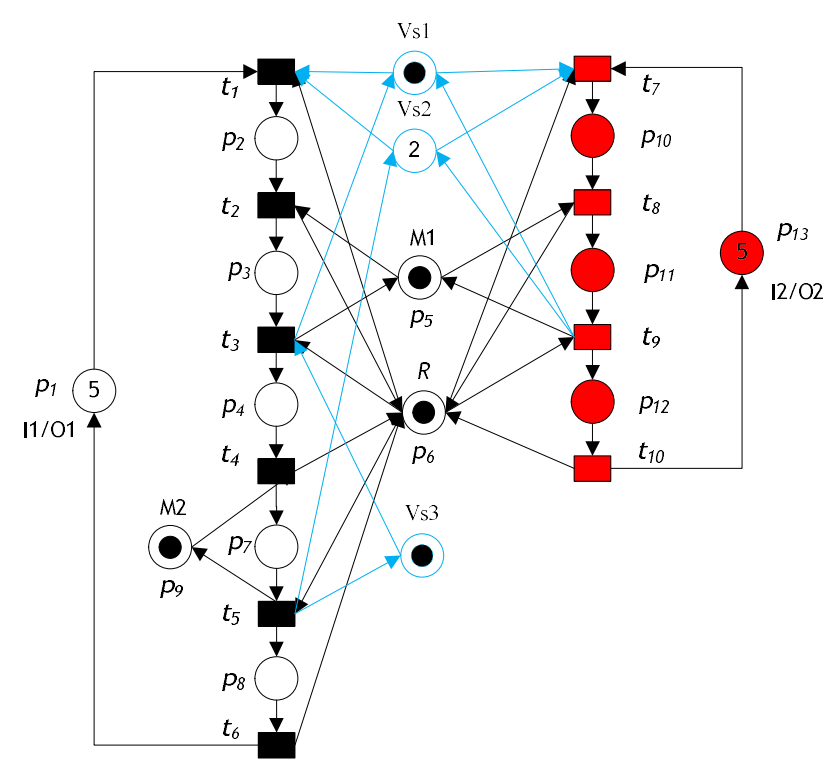

Figure 9. Controlled reconfigurable $S^{3} \mathrm{PR}$ net by addition of new product.

The following place invariants are accordingly generated:

$$
\begin{aligned}
& M\left(V_{S 1}\right)=M\left(p_{4}\right)+M\left(p_{5}\right)+M\left(p_{6}\right)+M\left(p_{8}\right)+M\left(p_{12}\right)+M\left(p_{16}\right)-1 \\
& M\left(V_{S 2}\right)=M\left(p_{5}\right)+M\left(p_{6}\right)+M\left(p_{8}\right)+M\left(p_{9}\right)+M\left(p_{12}\right)+M\left(p_{16}\right)-1 \\
& M\left(V_{S 3}\right)=M\left(p_{4}\right)+M\left(p_{5}\right)+M\left(p_{6}\right)+M\left(p_{8}\right)+M\left(p_{16}\right)+M\left(p_{17}\right)-1 \\
& M\left(V_{S 4}\right)=M\left(p_{5}\right)+M\left(p_{6}\right)+M\left(p_{8}\right)+M\left(p_{9}\right)+M\left(p_{16}\right)+M\left(p_{17}\right)-1 \\
M\left(V_{S 5}\right)= & M\left(p_{2}\right)+M\left(p_{6}\right)+M\left(p_{8}\right)+M\left(p_{9}\right)+M\left(p_{10}\right)+M\left(p_{12}\right)+M\left(p_{15}\right)+M\left(p_{16}\right)-1 \\
M\left(V_{S 6}\right)= & M\left(p_{2}\right)+M\left(p_{4}\right)+M\left(p_{6}\right)+M\left(p_{8}\right)+M\left(p_{10}\right)+M\left(p_{15}\right)+M\left(p_{16}\right)+M\left(p_{17}\right)-1 \\
M\left(V_{S 7}\right)= & M\left(p_{2}\right)+M\left(p_{6}\right)+M\left(p_{8}\right)+M\left(p_{9}\right)+M\left(p_{10}\right)+M\left(p_{15}\right)+M\left(p_{16}\right)+M\left(p_{17}\right)-1
\end{aligned}
$$

The current matrices $\mathcal{L}$ and $\mathcal{B}$ represent the Equations (38)-(44).

$$
\mathcal{L}=\left[\begin{array}{lllllllllllllllll}
0 & 0 & 0 & 1 & 1 & 1 & 0 & 1 & 0 & 0 & 0 & 1 & 0 & 0 & 0 & 1 & 0 \\
0 & 0 & 0 & 0 & 1 & 1 & 0 & 1 & 1 & 0 & 0 & 1 & 0 & 0 & 0 & 1 & 0 \\
0 & 0 & 0 & 1 & 1 & 1 & 0 & 1 & 0 & 0 & 0 & 0 & 0 & 0 & 0 & 1 & 1 \\
0 & 0 & 0 & 0 & 1 & 1 & 0 & 1 & 1 & 0 & 0 & 0 & 0 & 0 & 0 & 1 & 1 \\
0 & 1 & 0 & 0 & 0 & 1 & 0 & 1 & 1 & 1 & 0 & 1 & 0 & 0 & 1 & 1 & 0 \\
0 & 1 & 0 & 1 & 0 & 1 & 0 & 1 & 0 & 1 & 0 & 0 & 0 & 0 & 1 & 1 & 1 \\
0 & 1 & 0 & 0 & 0 & 1 & 0 & 1 & 1 & 1 & 0 & 0 & 0 & 0 & 1 & 1 & 1
\end{array}\right], \mathcal{B}=\left[\begin{array}{l}
1 \\
1 \\
1 \\
1 \\
1 \\
1 \\
1
\end{array}\right]
$$


The controller's net incidence matrix is calculated by Equation (12);

$$
\left[N_{V_{S}}\right]=\left[\begin{array}{cccccccccccccc}
-1 & 0 & 1 & 0 & 0 & 0 & -1 & 0 & 1 & 0 & -1 & 0 & 0 & 0 \\
-1 & 0 & 0 & 0 & 1 & 0 & -1 & 0 & 1 & 0 & -1 & 0 & 0 & 0 \\
-1 & 0 & 1 & 0 & 0 & 0 & -1 & 0 & 0 & 0 & 0 & 1 & 0 & 0 \\
-1 & 0 & 0 & 0 & 1 & 0 & -1 & 0 & 0 & 0 & 0 & 1 & 0 & 0 \\
0 & 0 & -1 & 0 & 1 & 0 & 0 & 0 & 0 & 0 & 0 & 0 & 0 & 0 \\
0 & 0 & 0 & 0 & 0 & 0 & 0 & 0 & -1 & 0 & 1 & 1 & 0 & 0 \\
0 & 0 & -1 & 0 & 1 & 0 & 0 & 0 & -1 & 0 & 1 & 1 & 0 & 0
\end{array}\right]
$$

The initial marking controllers are calculated as:

$M_{o}\left(V_{S 1}\right)=1, M_{o}\left(V_{S 2}\right)=2, M_{o}\left(V_{S 3}\right)=2, M_{o}\left(V_{S 4}\right)=3, M_{o}\left(V_{S 5}\right)=1, M_{o}\left(V_{S 6}\right)=1$, and $M_{o}\left(V_{S 7}\right)=2$.

The controlled reconfigurable net of $\left(N_{3}, M_{30}\right)$ is illustrated in Figure 10. The place and arcs of the controller are shown with blue lines.

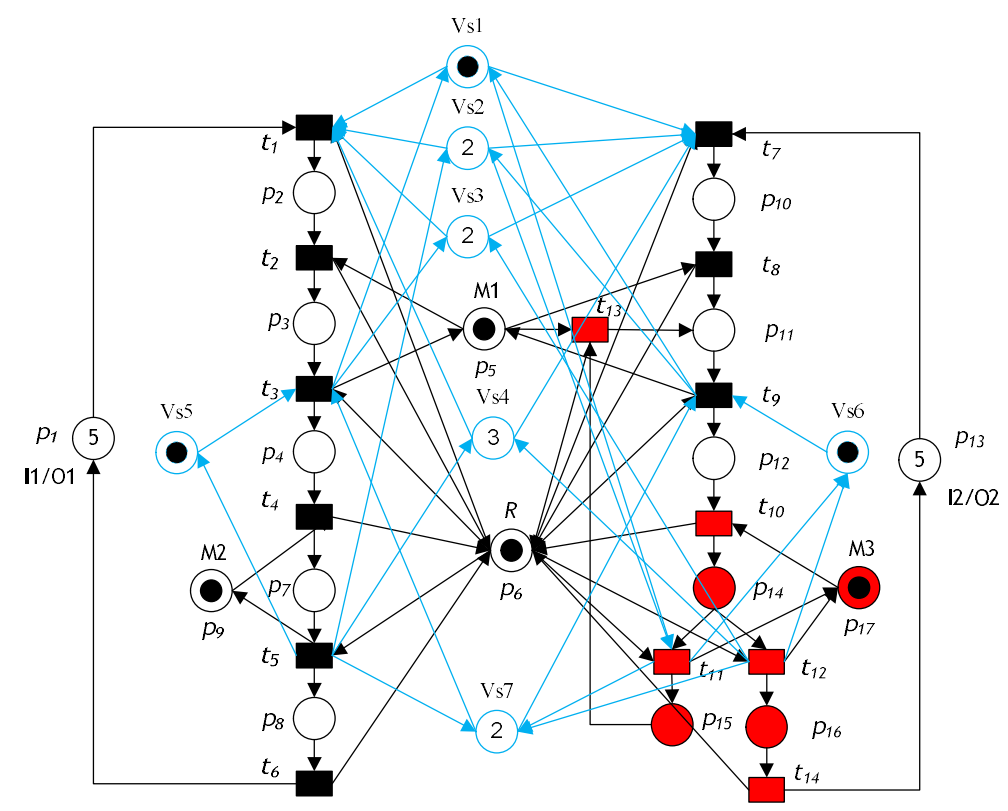

Figure 10. Controlled reconfigurable $S^{3} \mathrm{PR}$ net by rework.

Finally, reconsider the reconfigured $S^{3} \mathrm{PR}$ net by addition of a new robot $\left(N_{4}, M_{40}\right)$ illustrated in Figure 6c. The reconfigured net has 17 minimal siphons, ten of which $S_{1}-S_{10}$ that create monitors $V_{S 1^{-}}$ $V_{S 10}$, respectively, which are siphons $S_{1}=\left\{p_{2}, p_{6}, p_{6-1}, p_{8}, p_{9}\right\}, S_{2}=\left\{p_{4}, p_{5}, p_{6-1}, p_{8}, p_{11}\right\}, S_{3}=\left\{p_{5}, p_{6-1}, p_{8}\right.$, $\left.p_{9}, p_{11}\right\}, S_{4}=\left\{p_{4}, p_{5}, p_{6-1}, p_{6-2}, p_{8}, p_{12}, p_{16}\right\}, S_{5}=\left\{p_{4}, p_{5}, p_{6-1}, p_{6-2}, p_{8}, p_{16}, p_{17}\right\}, S_{6}=\left\{p_{5}, p_{6-1}, p_{6-2}, p_{8} p_{9}\right.$, $\left.p_{12}, p_{16}\right\}, S_{7}=\left\{p_{5}, p_{6-1}, p_{6-2}, p_{8}, p_{9}, p_{16}, p_{17}\right\}, S_{8}=\left\{p_{3}, p_{5}, p_{6-2}, p_{12}, p_{16}\right\}, S_{9}=\left\{p_{3}, p_{5}, p_{6-2}, p_{16}, p_{17},\right\}$, and $S_{10}$ $=\left\{p_{6-2}, p_{10}, p_{15}, p_{16}, p_{17}\right\}$. The current matrices $\mathcal{L}$ and $\mathcal{B}$ are expressed as:

$$
\mathcal{L}=\left[\begin{array}{llllllllllllllllll}
0 & 1 & 0 & 0 & 0 & 1 & 0 & 0 & 1 & 1 & 0 & 0 & 0 & 0 & 0 & 0 & 0 & 0 \\
0 & 0 & 0 & 1 & 1 & 1 & 0 & 0 & 1 & 0 & 0 & 1 & 0 & 0 & 0 & 0 & 0 & 0 \\
0 & 0 & 0 & 0 & 1 & 1 & 0 & 0 & 1 & 1 & 0 & 1 & 0 & 0 & 0 & 0 & 0 & 0 \\
0 & 0 & 0 & 1 & 1 & 1 & 1 & 0 & 1 & 0 & 0 & 0 & 1 & 0 & 0 & 0 & 1 & 0 \\
0 & 0 & 0 & 1 & 1 & 1 & 1 & 0 & 1 & 0 & 0 & 0 & 0 & 0 & 0 & 0 & 1 & 1 \\
0 & 0 & 0 & 0 & 1 & 1 & 1 & 0 & 1 & 1 & 0 & 0 & 1 & 0 & 0 & 0 & 1 & 0 \\
0 & 0 & 0 & 0 & 1 & 1 & 1 & 0 & 1 & 1 & 0 & 0 & 0 & 0 & 0 & 0 & 1 & 1 \\
0 & 0 & 1 & 0 & 1 & 0 & 1 & 0 & 0 & 0 & 0 & 0 & 1 & 0 & 0 & 0 & 1 & 0 \\
0 & 0 & 1 & 0 & 1 & 0 & 1 & 0 & 0 & 0 & 0 & 0 & 0 & 0 & 0 & 0 & 1 & 1 \\
0 & 0 & 0 & 0 & 0 & 0 & 1 & 0 & 0 & 0 & 1 & 0 & 0 & 0 & 0 & 1 & 1 & 1
\end{array}\right], \mathcal{B}=\left[\begin{array}{l}
1 \\
1 \\
1 \\
1 \\
1 \\
1 \\
1 \\
1 \\
1 \\
1
\end{array}\right]
$$


The controller's net incidence matrix is calculated by Equation (12);

$$
\left[N_{V_{S}}\right]=\left[\begin{array}{ccccccccccccccc}
0 & 0 & -1 & 0 & 1 & 0 & 0 & 0 & 0 & 0 & 0 & 0 & 0 & 0 & 0 \\
-1 & 0 & 1 & 0 & 0 & 0 & 0 & 0 & 0 & 0 & 0 & 0 & 0 & 0 & 0 \\
-1 & 0 & 0 & 0 & 1 & 0 & 0 & 0 & 0 & 0 & 0 & 0 & 0 & 0 & 0 \\
-1 & 0 & 1 & 0 & 0 & 0 & -1 & 0 & 1 & 0 & -1 & 0 & 0 & 0 & 0 \\
-1 & 0 & 1 & 0 & 0 & 0 & -1 & 0 & 0 & 0 & 0 & 1 & 1 & 0 & 0 \\
-1 & 0 & 0 & 0 & 1 & 0 & -1 & 0 & 1 & 0 & -1 & 0 & 0 & 0 & 0 \\
-1 & 0 & 0 & 0 & 1 & 0 & -1 & 0 & 0 & 0 & 0 & 1 & 1 & 0 & 0 \\
0 & 0 & 0 & 0 & 0 & 0 & -1 & 0 & 1 & 0 & -1 & 0 & 0 & 0 & 0 \\
0 & 0 & 0 & 0 & 0 & 0 & -1 & 0 & 0 & 0 & 0 & 1 & 1 & 0 & 0 \\
0 & 0 & 0 & 0 & 0 & 0 & 0 & 0 & -1 & 0 & 1 & 1 & 1 & 0 & 0
\end{array}\right]
$$

The initial marking controllers are calculated as $M_{o}\left(V_{S 1}\right)=1, M_{o}\left(V_{S 2}\right)=1, M_{o}\left(V_{S 3}\right)=2, M_{o}\left(V_{S 4}\right)=$ $2, M_{o}\left(V_{S 5}\right)=3, M_{o}\left(V_{S 6}\right)=3, M_{o}\left(V_{S 7}\right)=4, M_{o}\left(V_{S 8}\right)=1, M_{o}\left(V_{S 9}\right)=2$, and $M_{o}\left(V_{S 10}\right)=1$.

The controlled reconfigurable net of $\left(N_{4}, M_{40}\right)$ is illustrated in Figure 11. The place and arcs of the controller are shown with blue lines.

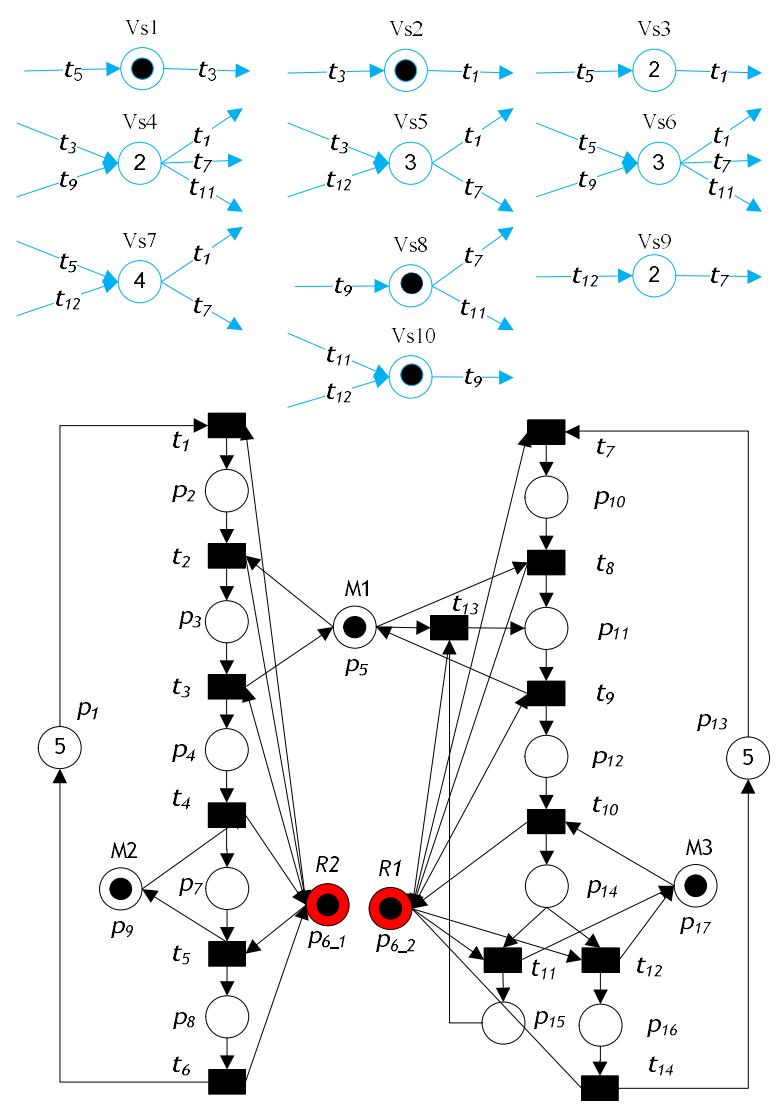

Figure 11. Controlled reconfigurable $S^{3} P R$ net by rework.

\section{Behavioral and Quantitative Analysis of Reconfigurable $S^{3}$ PR Net}

\subsection{Liveness}

Liveness is one of the most important issues in reconfigurable manufacturing systems with dynamic changes. Conversely, in these systems, deadlock is usually unwanted. When a system is not live, tasks could never be performed because of local or global deadlocks. Liveness of a transition means that, irrespective of the current state of the net, it can always eventually fire. 
Theorem 7. The controlled reconfigurable $S^{3} P R$ net $\left(N_{R C}, M_{R C o}\right)$ with $N_{R C}=\left(P_{R C}, T_{R C}, F_{R C}, W_{R C}, M_{R C o}\right.$, $\left.K_{R C}\right)$ is live.

Proof. All transitions $T_{R C}$ in $\left(N_{R C}, M_{R C o}\right)$ must be proven to be live. There is no unmarked siphon, $p \in S$. $p$ is marked at marking $M$ and satisfies $\sum_{p \in S} M(p) \geq 1$, since all $t \in T_{R C}$ are live. For all $t \in T_{R C}$, if for all $p \in \bullet^{\bullet} t, M_{R C o}(p)>0$, then $t$ can fire in any case. Therefore, the controlled reconfigurable $S^{3} P R$ net $\left(N_{R C}, M_{R C o}\right)$ is live.

To demonstrate the liveness of a reconfigurable $S^{3} \mathrm{PR}$ net, consider the model illustrated in Figure 9. Its reachability graph with all model markings is illustrated in Figure 12 and it is apparent that all transitions are live, which means that the system is live.

\subsection{Boundedness}

The boundedness is associated with a place, indicating that the number of tokens in a place never exceeds a certain number. This means that there is no overflow in a place.

Theorem 8. Let a reconfigurable $S^{3} P R$ net $\left(N_{R C}, M_{R C o}\right)$ with $N_{R C}=\left(P_{R C}, T_{R C}, F_{R C}, W_{R C}, M_{R C o}, K_{R C}\right)$ be a controlled net. Then $\left(N_{R C}, M_{R C o}\right)$ is bounded.

Proof. Theorem 7 proves that the net $\left(N_{R C}, M_{R C o}\right)$ is live. Therefore, the boundedness can be established by checking if the net $\left(N_{R C}, M_{R C_{0}}\right)$ is well constructed, behaved, and controlled. The resulting net $\left(N_{R C}, M_{R C_{0}}\right)$ maintains the boundedness as the net is well constructed, behaved and has a finite reachability set.

To demonstrate the boundedness of a controlled reconfigurable $\mathrm{S}^{3} \mathrm{PR}$ net, consider the net illustrated in Figure 9. Its reachability graph is illustrated in Figure 12. It is obvious that markings reachable from initial marking are five-bounded, which indicates that the system is bounded.

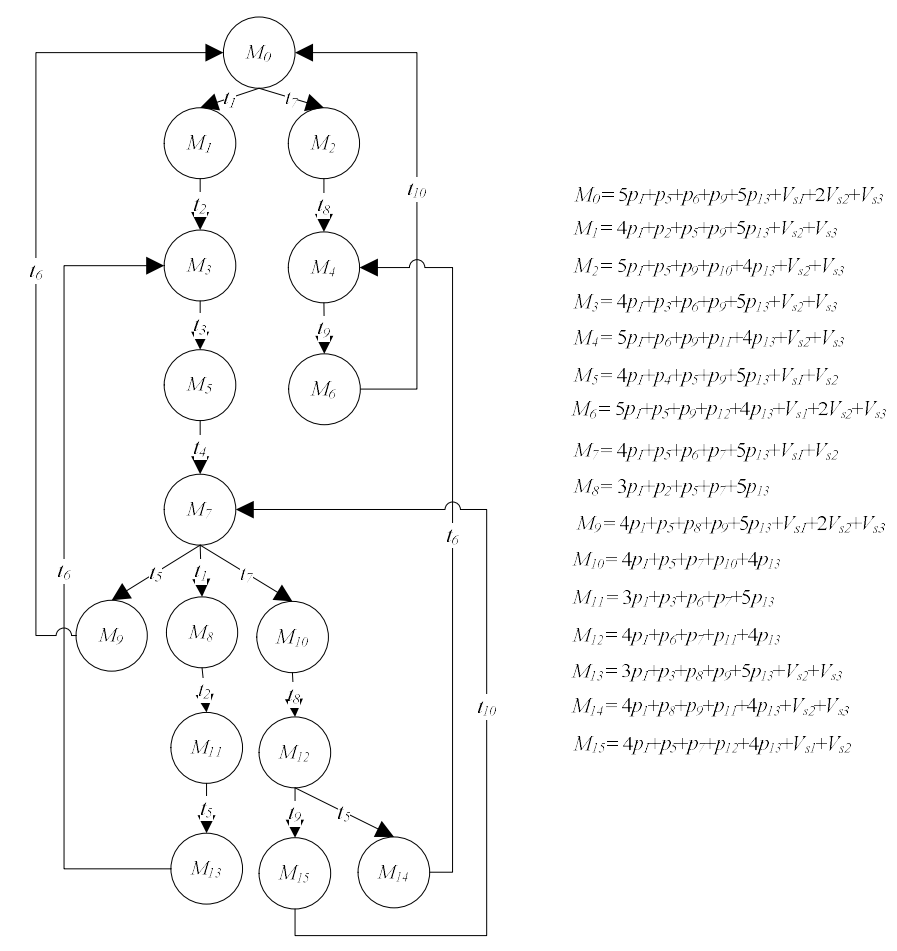

Figure 12. Reachable markings of a controlled reconfigurable $S^{3} \mathrm{PR}$ net, as illustrated in Figure 8. 


\subsection{Reversibility}

Reversibility means that a system can always return to its initial marking. A controlled reconfigurable $S^{3} P R$ Petri net model $\left(N_{R C}, M_{R C o}\right)$ is reversible if for each marking $M \in R\left(N_{R C}\right.$, $M_{R C o}$ ), initial marking $M_{R C o}$ is reachable from $M$.

Theorem 9. Let a reconfigurable $S^{3} P R$ net $\left(N_{R C}, M_{R C o}\right)$ with $N_{R C}=\left(P_{R C}, T_{R C}, F_{R C}, W_{R C}, M_{R C o}, K_{R C}\right)$ be a live and controlled net. $N_{R C}$ is reversible if for each marking $M \in R\left(N_{R C}, M_{R C_{0}}\right)$, initial marking $M_{R C_{0}}$ is reachable from $M, M$ and $M_{R C o}$ satisfying all place invariants and $M$ marks each trap of $N_{R C}$.

Proof. Suppose that $M$ is reachable. Then there exists a finite transition sequence $\delta=t_{1} t_{2} t_{3} \ldots t_{n}$ that can be fired, and markings $M_{1}, M_{2}, M_{3}, \ldots$, and $M_{n-1}$ are such that $M_{R C o}\left[t_{1}\right\rangle M_{1}\left[t_{2}\right\rangle M_{2}\left[t_{3}\right\rangle M_{3} \ldots$ $M_{n-1}\left[t_{n}\right\rangle M$, expressed as $M_{R C o}[\delta\rangle M$, agrees with the state equation $M=M_{R C o}+\left[N_{R C}\right] \vec{\delta}$. In addition, $M$ and $M_{R C_{o}}$ satisfy all place invariants, $I^{T} \cdot M=I^{T} \cdot M_{R C_{0}}$. Therefore, we can say that $M_{R C_{o}}$ is the home marking of the net $\left(N_{R C}, M_{R C o}\right), M$ is reachable from $M_{R C o}$, and we get $M_{R C_{0}} \stackrel{\vec{\delta}}{\rightarrow} M$. Thus, the reconfigurable $S^{3} \mathrm{PR}$ net $\left(N_{R C}, M_{R C o}\right)$ is reversible.

To demonstrate the reversibility of a controlled reconfigurable $\mathrm{S}^{3} \mathrm{PR}$ net, consider the model illustrated in Figure 8. Its reachability graph is illustrated in Figure 13. In the net shown in Figure 8, there are seven minimal place invariants: $I_{1}=p_{3}+p_{5}, I_{2}=p_{2}+p_{3}+p_{10}, I_{3}=p_{7}+p_{9}, I_{4}=p_{4}+p_{7}+p_{11}$, $I_{5}=p_{2}+p_{3}+p_{4}+p_{7}+p_{12}, I_{6}=p_{2}+p_{4}+p_{6}+p_{8}, I_{7}=p_{1}+p_{2}+p_{3}+p_{4}+p_{7}+p_{8}$, since $\forall \mathrm{i} \in\{1,2,3,4,5,6,7\}$, $I_{i}^{T} \cdot\left[N_{R C}\right]=0^{T} \cdot M_{6} \in R\left(N_{R C}, M_{R C_{0}}\right), I_{1}^{T} \cdot M_{6}=I_{1}^{T} \cdot M_{R C_{0}}=M_{6}\left(p_{3}\right)+M_{6}\left(p_{5}\right)=M_{R C_{0}}\left(p_{3}\right)+M_{R C_{0}}\left(p_{5}\right)=1$. The net has a unique T-invariant $J=t_{1}+t_{2}+t_{3}+t_{4}+t_{5}+t_{6}$ and the transition sequence $\delta=t_{1} t_{2} t_{3} t_{4} t_{5} t_{6}$ is firable. As a result, $M_{R C o}\left[t_{1}\right\rangle M_{1}\left[t_{2}\right\rangle M_{2}\left[t_{3}\right\rangle M_{3}\left[t_{4}\right\rangle M 4\left[t_{5}\right\rangle M 6\left[t_{6}\right\rangle M_{R C o}$. Therefore, the reconfigurable $\mathrm{S}^{3} \mathrm{PR}$ net $\left(N_{R C}, M_{R C_{0}}\right)$ is live, bounded, and reversible.

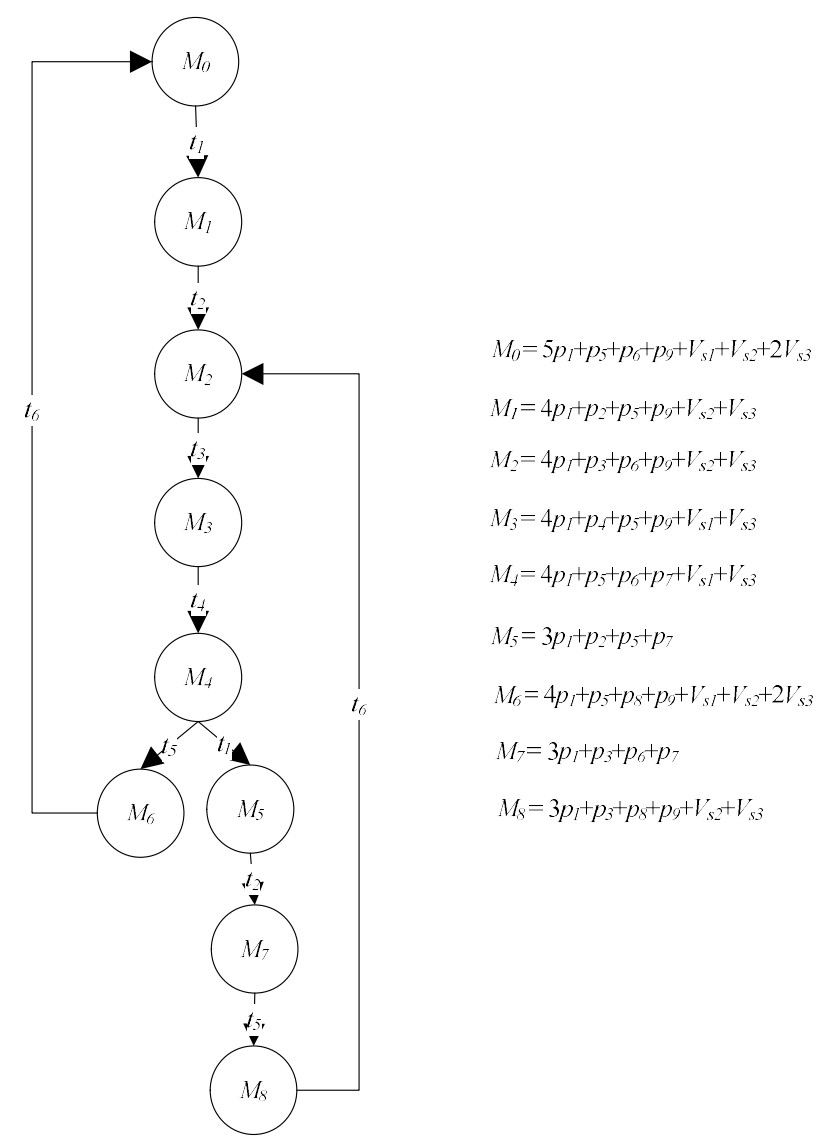

Figure 13. Reachable markings of a controlled reconfigurable $S^{3} \mathrm{PR}$ net, as illustrated in Figure 7. 


\subsection{Computational Complexity}

Algorithm 1 is used to design a reconfigurable $S^{3} P R$ net with $N_{R}=\left(\left(N, M_{0}\right), \mathcal{R}\right)$. In addition, Algorithm 2 computes the control places to a reconfigurable $S^{3} P R$ net with $N_{R}=\left(\left(N, M_{0}\right), \mathcal{R}\right)$.

Theorem 10. Given a reconfigurable $S^{3} P R$ net with $N_{R}=\left(\left(N, M_{0}\right), \mathcal{R}\right)$, where $N_{R}$ with $N_{k}=\left(P_{C k}, T_{k}, F_{k}, W_{k}\right.$, $\left.M_{k o}, K_{k}\right)$, the time complexity of Algorithm 1 is polynomial.

Proof. Let $N_{k}$ be states in $N_{R}$ with $N_{k}=\left(P_{C k}, T_{k}, F_{k}, W_{k}, M_{k o}, K_{k}\right), \mathcal{R}=\left\{r r_{1}, r r_{2}, r r_{3}, \ldots, r r_{k}\right\}, r r_{k}=\left\{L_{k}, R_{k}\right.$, $\left.\varphi_{k}, \varphi_{k}, \varphi_{k}{ }^{\bullet}\right\}$, and net $\left(N_{R}, M_{R o}\right)$ be the obtained reconfigurable $S^{3} P R$ net. Let $x$ be the cardinality of $\mathcal{R}$, i.e., $|\mathcal{R}|=x$. The "While" loop is executed $x$ times to design state $N_{k}$ in a reconfigurable $\mathrm{S}^{3} \mathrm{PR}$ net $\left(N_{R}\right.$, $\left.M_{R o}\right)$. Therefore, in the worst case, the computational complexity of algorithm 1 is $\mathrm{O}(x)$. Thus, the computational complexity of the Algorithm 1 has polynomial time complexity.

Theorem 11. Given a reconfigurable $S^{3} P R$ net with $N_{R}=\left(\left(N, M_{o}\right), \mathcal{R}\right)$, where $N_{R}$ with $N_{k}=\left(P_{C k}, T_{k}, F_{k}, W_{k}\right.$, $\left.M_{k o}, K_{k}\right)$, the time complexity of Algorithm 2 is polynomial.

Proof. Algorithm 2 is used to design a control place $V_{S}$ to each minimal siphon $S, V_{S} \bullet{ }^{\bullet} S$ in each state $N_{k}$ in a reconfigurable $S^{3} P R$ net $\left(N_{R}, M_{R o}\right)$ to achieve the liveness of net $\left(N_{R}, M_{R o}\right)$. Obviously, each $V_{S}$ is associated with the minimal siphon $S$ in net $\left(N_{k}, M_{k o}\right)$. Let $x$ be the cardinality of $\mathcal{R}$, i.e., $|\mathcal{R}|=$ $x$. Let $y$ be the number of minimal siphons $S$ (denoted as $S^{\prime}$ ) that requires $V_{S}$ i.e., $\left|S^{\prime}\right|=y$. The "While" loop is executed $x$ times to design state $N_{k}$ in reconfigurable $S^{3} \mathrm{PR}$ net $\left(N_{R}, M_{R o}\right)$. The "FOR loop" loop is executed $y$ times to design $V_{S}$ for the $S^{\prime}$ in $\left(N_{k}, M_{k o}\right)$. Therefore, the computational complexity of Algorithm 2 is $\mathrm{O}(x y)$. Thus, the computational complexity of the Algorithm 2 has polynomial time complexity.

\subsection{GPENSIM Code and Validation}

We coded the developed approach using the GPenSIM tool $[6,42]$ to verify and validate it and compared the developed code with the studies by Ezpeleta et al. [43], Li and Zhou [44], and Kaid et al. [6]. There were three files generated: (1) the Petri net definition file (PDF) that represents the static model by stating the sets of places, transitions, and arcs, (2) the common processor file (COMMON_PRE file) that represents the conditions for activation of the enabling fire transitions, and (3) the main simulation file (MSF) that calculates the results of the simulation. The developed approach was implemented on MATLAB R2015a. A PC with Windows 10, 64-bit and Intel(R) Core (TM) i7-4702MQ CPU @ 2.20 GHz, 16 GB RAM.

Simulation leads to a better time performance in the designed model including total throughput time (total time in system), total throughput, and utilization of the robots and machines. Consider the model illustrated in Figure 8. The simulation was undertaken for $480 \mathrm{~min}$. The results summarized in Table 1 were obtained after simulation in MATLAB. Table 1 shows the results for the time performance criteria mentioned above. All methods achieve approximately the same values for the utilization of resources as illustrated in Figure 14. In addition, the proposed method, as illustrated in Figure 14, can achieve approximately the same values with other techniques for throughput. In term of throughput time of Part A, the proposed method can achieve approximately the same values with other techniques as illustrated in Figure 14. Therefore, the proposed method is valid, sufficiently accurate results can be obtained and other cases can be applied.

Table 1. Time performance comparison with the existing methods.

\begin{tabular}{ccccc}
\hline Performance & Ezpeleta et al. [43] & Li and Zhou [44] & Kaid et al. [6] & The Proposed Method \\
\hline M1 utilization (\%) & 29.05 & 29.05 & 29.60 & 29.05 \\
M2 utilization (\%) & 29.61 & 29.61 & 30.50 & 29.61 \\
R1 utilization (\%) & 48.04 & 48.04 & 47.56 & 48.04 \\
Throughput (parts) & 34 & 34 & 34 & 34 \\
Throughput time (min/part) & 14.12 & 14.12 & 14.12 & 14.12 \\
\hline
\end{tabular}




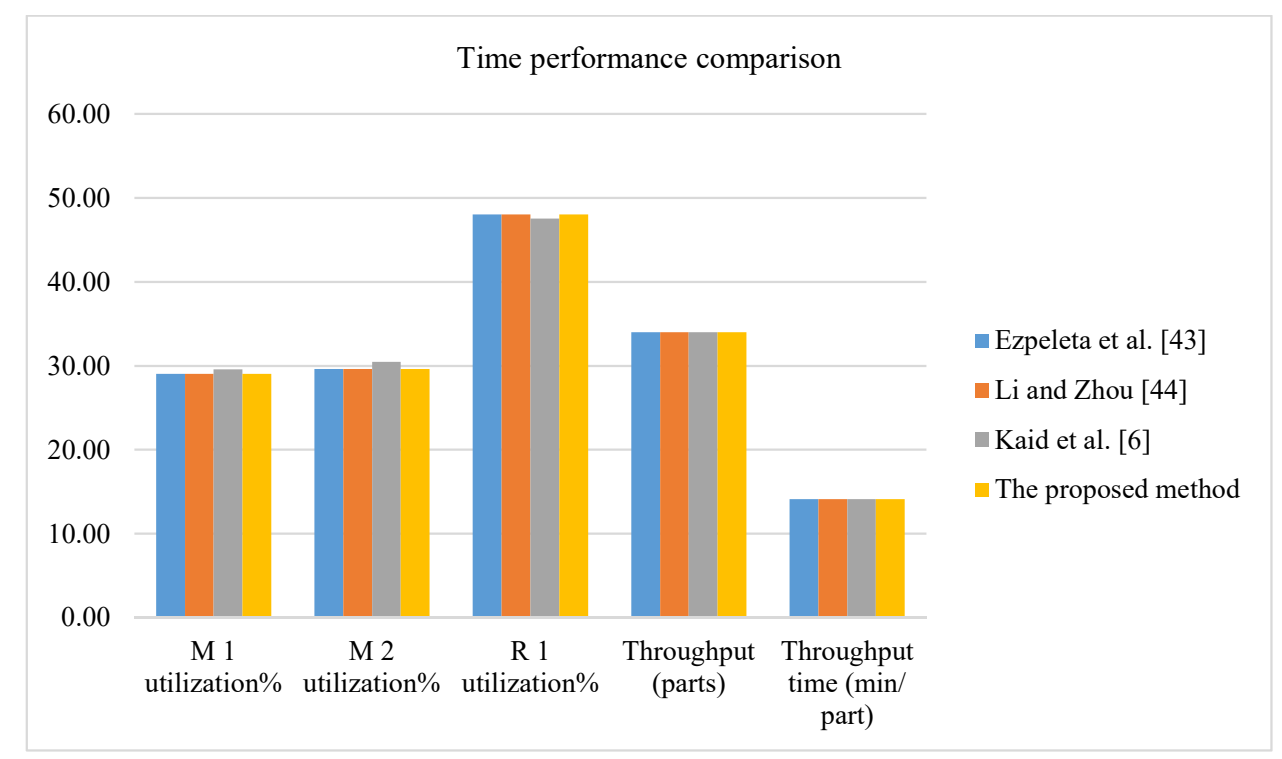

Figure 14. Comparison of the proposed method with the existing methods.

\section{Numerical Example}

In this section, an example is used to present the application of the proposed approach. Consider an AMS example illustrated in Figure 15a. Its Petri net model is given in $[6,7,15,22,45,46]$. The system consists of four machines M1-M4 for processing parts; two robots R1 and R2 for loading and unloading parts. Each machine (robot) can process (hold) one part at a time. There are two input buffers I1 and I2 and two output buffers $\mathrm{O} 1$ and $\mathrm{O} 2$. Two raw part types, $\mathrm{A}$ and $\mathrm{B}$, are considered to be processed in the system. Figure $15 \mathrm{~b}$ shows the operation sequences of the two raw part types. The $\mathrm{S}^{3} \mathrm{PR}$ net of this AMS example is illustrated in Figure 16. It comprises 19 places and 14 transitions. The places can be defined as the following set partitions: $P_{A}=\left\{p_{2}, p_{3}, \ldots, p_{12}\right\}, P_{R}=\left\{p_{13}, p_{14}, \ldots, p_{18}\right\}$, and $P^{0}=\left\{p_{1}, p_{19}\right\}$. The $S^{3} P R$ net contains 282 reachable markings.

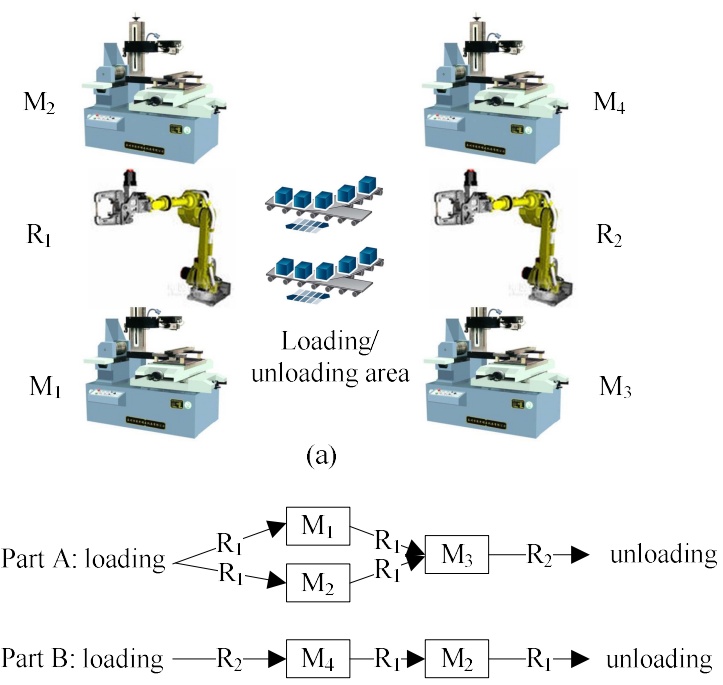

(b)

Figure 15. (a) An AMS example and (b) production sequence. 


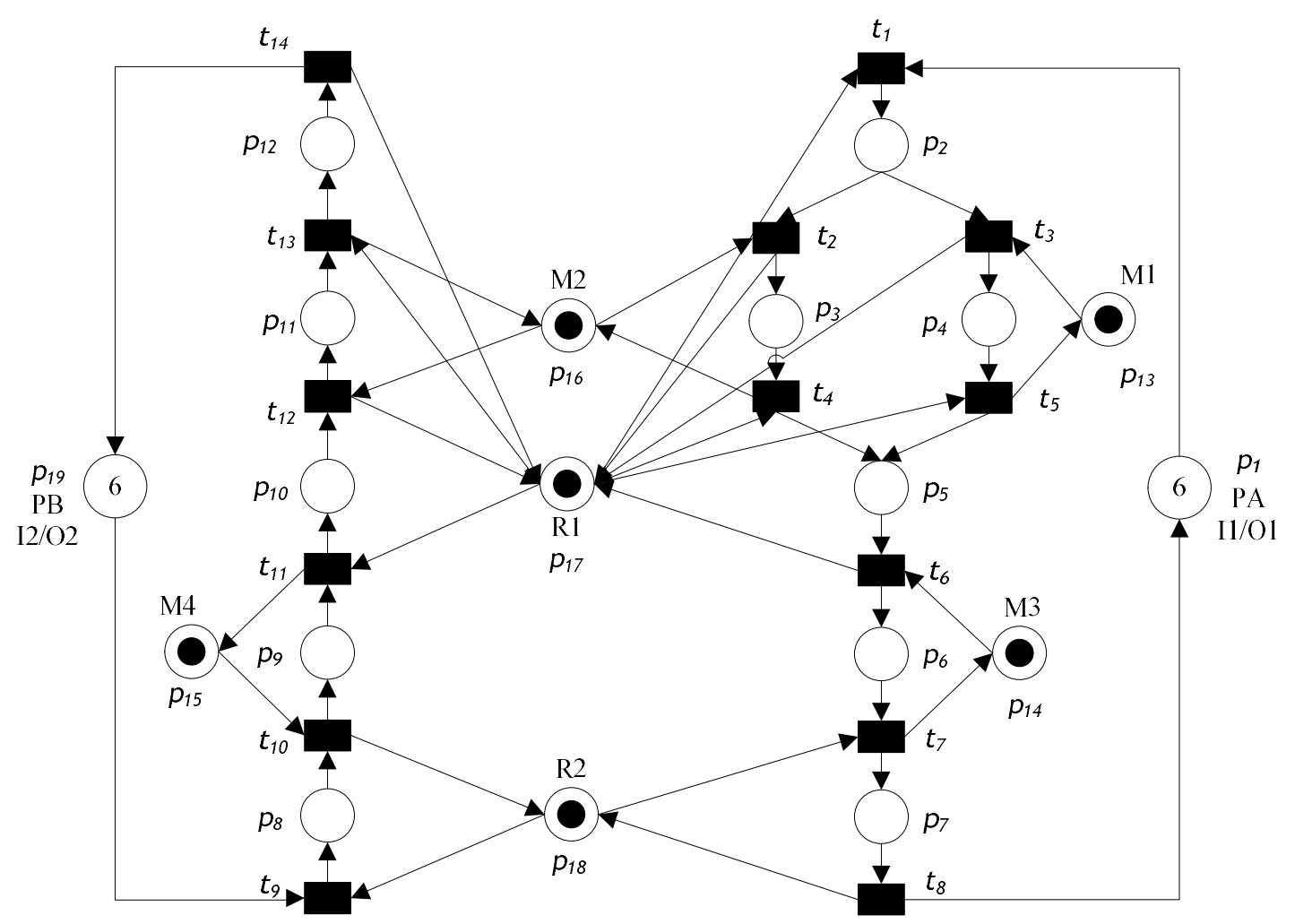

Figure 16. $\mathrm{S}^{3} \mathrm{PR}$ net $\left(N_{0}, M_{0}\right)$ of the AMS illustrated in Figure 13a.

Suppose that the first configuration of the system involves removing old machine. In this case, an old machine $\mathrm{M} 1$ is removed from the system $\left(N_{o}, M_{o}\right)$. To model the removed machine by using the synthesis procedure of Algorithm 1, we construct a configuration as a rewriting rule $\mathcal{R}=\left\{r r_{1}\right\}$ with $r r_{1}=\left\{L_{1}, R_{1}, \varphi_{1}, \varphi^{\bullet}, \varphi_{1} \bullet\right.$, where $L_{1}$ and $R_{1}$ are illustrated in Figures $17 \mathrm{a}$ and 17b, respectively. In addition, we have $\xi_{1}: N_{1} \rightarrow N_{o}, \varphi_{1}=\left(\left\{p_{2}, p_{3}, p_{4}, p_{5}, p_{13}, p_{16}, p_{17}\right\},\left\{t_{1}, t_{2}, t_{3}, t_{4}, t_{5}, t_{6}\right\}\right), \bullet \varphi_{1}=\left(\left\{L_{1} . t_{1}\right\},\left\{R_{1} . t_{1}\right\}\right)$, and $\varphi_{1} \cdot=\left(\left\{L_{1} \cdot t_{6}\right\},\left\{R_{1} \cdot t_{6}\right\}\right)$. The second configuration includes adding new product. If a new product (part $\mathrm{C}$ ) is assigned to a system, which indicates that a new operation sequence is assigned and the system requires an adjustment to its Petri net model structure. To model the addition of new product by using the synthesis procedure of Algorithm 1, we construct a configuration as a rewriting rule $\mathcal{R}=\left\{r r_{2}\right\}$ with $r r_{2}=\left\{L_{2}, R_{1}, \varphi_{2}, \bullet \varphi_{2}, \varphi_{2} \bullet\right\}$, where $L_{2}$ and $R_{2}$ are illustrated in Figures 18a and 18b, respectively. Moreover, we have $\xi_{2}: N_{2} \rightarrow N_{1}, \varphi_{2}=\left(\left\{p_{15}, p_{17}, p_{20}, p_{21}, p_{22}, p_{23}\right\},\left\{t_{15}, t_{16}, t_{17}, t_{18}\right\}\right), \varphi^{\bullet} \varphi_{2}=$ $\left(\left\{L_{2} \cdot p_{15}, L_{2} \cdot p_{17}\right\},\left\{R_{2} \cdot t_{15}\right\}\right)$, and $\varphi_{2}{ }^{\bullet}=\left(\left\{L_{2} \cdot p_{15}, L_{2} \cdot p_{17}\right\},\left\{R_{2} \cdot t_{18}\right\}\right)$.

The third system configuration involves rework. In this scenario, a part can be inspected after all operations have been completed. By using the proposed Algorithm 1, the production operations of the reworked part can be exactly and easily modeled by considering rework operations as alternative sequences. Suppose that an inspection machine M5 is added to a system and that part A is processed in M1 and M3. Then, part A is moved to an M5 by Robot 2 to check if there are defects in part A. If part A performs properly, then it will leave the system by Robot 2. Otherwise, if part A has defects, rework is needed, and part A is moved to M3 by Robot 2 . To model the rework operation by using the synthesis procedure of Algorithm 1, we construct a configuration as a rewriting rule $\mathcal{R}=\left\{r r_{3}\right\}$ with $r r_{3}$ $=\left\{L_{3}, R_{3}, \varphi_{3},{ }^{\bullet} \varphi_{3}, \varphi_{3} \bullet\right\}$, where $L_{3}$ and $R_{3}$ are illustrated in Figures 19a and 19b, respectively, $\xi_{3}: N_{3}$ $\rightarrow N_{2}, \varphi_{3}=\left(\left\{p_{6}, p_{7}, p_{14}, p_{18}, p_{24}, p_{25}, p_{26}, p_{27}\right\},\left\{t_{6}, t_{7}, t_{8}, t_{19}, t_{20}, t_{21}, t_{22}\right\}\right),{ }^{\bullet} \varphi_{3}=\left(\left\{L_{3} . t_{6}\right\},\left\{R_{3} . t_{6}\right\}\right)$, and $\varphi_{3} \bullet$ $=\left(\left\{L_{3} . t_{8}\right\},\left\{R_{3} . t_{21}\right\}\right)$. The Specifications of $S^{3} P R$ net illustrated in Figure 16 under changeable control specifications are shown in Table 2. In addition, the required monitors using Algorithm 2 of the system illustrated in Figure 16 under changeable control specifications are shown in Table 3. 


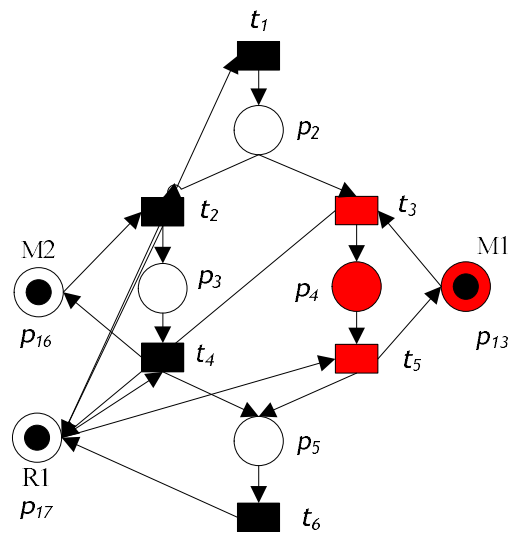

(a)

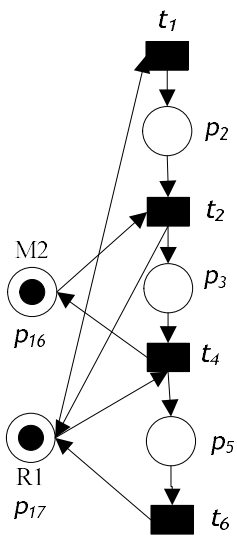

(b)

Figure 17. A reconfigured $S^{3} P R$ net by removing a machine. (a) Left hand side net $L$. (b) Right hand side net $R$.

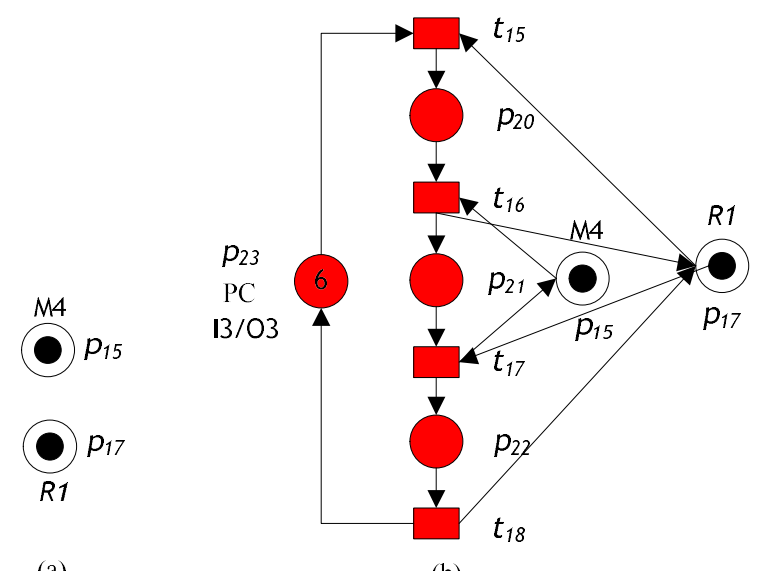

(a)

(b)

Figure 18. A reconfigured $S^{3} P R$ net by adding a product. (a) Left hand side net $L$. (b) Right hand side net $R$.
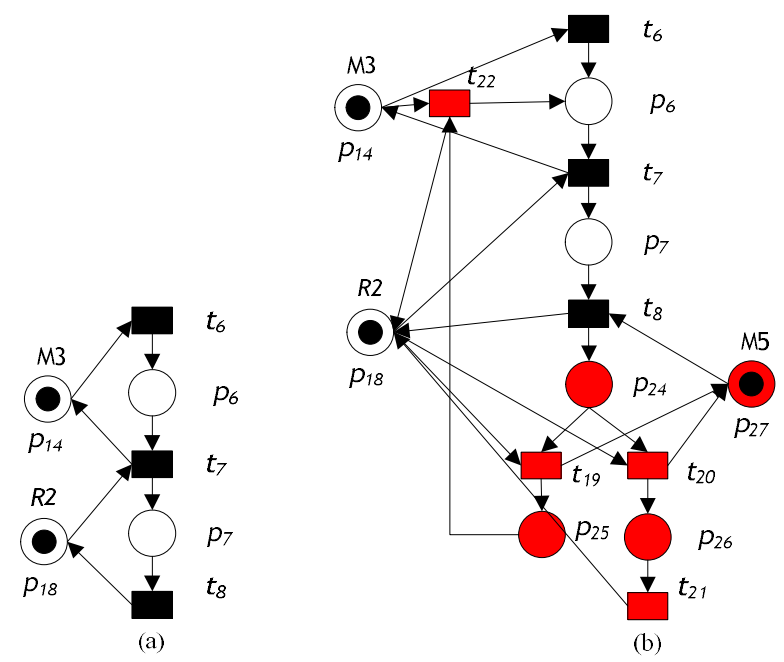

Figure 19. A reconfigured $S^{3} P R$ net by rework. (a) Left hand side net $L$. (b) Right hand side net $R$. 
Table 2. The Specifications of $S^{3} P R$ net illustrated in Figure 16 under configurations.

\begin{tabular}{ccccc}
\hline \multirow{2}{*}{ Parameter } & \multicolumn{4}{c}{ Configuration } \\
\cline { 2 - 5 } & $\begin{array}{c}\text { An Initial S } \\
\text { Net }\end{array}$ & $\begin{array}{c}\text { Removal of an } \\
\text { Old Machine }\end{array}$ & $\begin{array}{c}\text { Addition of a New } \\
\text { Product }\end{array}$ & Rework \\
\hline No. of monitors & 5 & 3 & 5 & 10 \\
No. of arcs & 21 & 12 & 26 & 48 \\
Liveness & Live & Live & Live & Live \\
Boundedness & Bounded & Bounded & Bounded & Bounded \\
Reversibility & Reversible & Reversible & Reversible & Reversible \\
\hline
\end{tabular}

Table 3. Required monitors using Algorithm 2 of the system illustrated in Figure 16 under configurations.

\begin{tabular}{|c|c|c|c|c|c|}
\hline Configuration & i & Siphon & $\cdot V_{S i}$ & $V_{S i}^{\bullet}$ & $M_{R C o}\left(V_{s i}\right)$ \\
\hline \multirow{5}{*}{ An initial $S^{3} P R$ net } & 1 & $S_{1}$ & $t_{7}, t_{13}$ & $t_{1}, t_{9}$ & 5 \\
\hline & 2 & $S_{2}$ & $t_{4}, t_{5}, t_{13}$ & $t_{1}, t_{11}$ & 2 \\
\hline & 3 & $S_{3}$ & $t_{7}, t_{13}$ & $t_{1}, t_{9}$ & 4 \\
\hline & 4 & $S_{4}$ & $t_{7}, t_{11}$ & $t_{1}, t_{9}$ & 3 \\
\hline & 5 & $S_{5}$ & $t_{4}, t_{13}$ & $t_{2}, t_{11}$ & 1 \\
\hline \multirow{3}{*}{$\begin{array}{l}\text { Removal of an old } \\
\text { machine }\end{array}$} & 1 & $S_{1}$ & $t_{4}, t_{13}$ & $t_{1}, t_{9}$ & 1 \\
\hline & 2 & $S_{2}$ & $t_{7}, t_{11}$ & $t_{4}, t_{9}$ & 3 \\
\hline & 3 & $S_{3}$ & $t_{7}, t_{13}$ & $t_{1}, t_{9}$ & 4 \\
\hline \multirow{5}{*}{$\begin{array}{l}\text { Addition of a new } \\
\text { product }\end{array}$} & 1 & $S_{1}$ & $t_{11}, t_{17}$ & $t_{10}, t_{15}$ & 1 \\
\hline & 2 & $S_{2}$ & $t_{4}, t_{13}, t_{17}$ & $t_{1}, t_{10}, t_{15}$ & 2 \\
\hline & 3 & $S_{3}$ & $t_{4}, t_{13}$ & $t_{1}, t_{9}$ & 1 \\
\hline & 4 & $S_{4}$ & $t_{7}, t_{11}, t_{17}$ & $t_{4}, t_{9}, t_{15}$ & 3 \\
\hline & 5 & $S_{5}$ & $t_{7}, t_{13}, t_{17}$ & $t_{1}, t_{9}, t_{15}$ & 4 \\
\hline \multirow{10}{*}{ Rework } & 1 & $S_{1}$ & $t_{7}$ & $t_{6}, t_{19}$ & 1 \\
\hline & 2 & $S_{2}$ & $t_{20}$ & $t_{6}$ & 2 \\
\hline & 3 & $S_{3}$ & $t_{6}, t_{20}$ & $t_{7}$ & 1 \\
\hline & 4 & $S_{4}$ & $t_{7}, t_{11}, t_{17}$ & $t_{4}, t_{9}, t_{15}, t_{19}$ & 3 \\
\hline & 5 & $S_{5}$ & $t_{7}, t_{13}, t_{17}$ & $t_{1}, t_{9}, t_{15}, t_{19}$ & 4 \\
\hline & 6 & $S_{6}$ & $t_{11}, t_{17}, t_{20}$ & $t_{4}, t_{9}, t_{15}$ & 4 \\
\hline & 7 & $S_{7}$ & $t_{13}, t_{17}, t_{20}$ & $t_{1}, t_{9}, t_{15}$ & 5 \\
\hline & 8 & $S_{8}$ & $t_{11}, t_{17}$ & $t_{8}, t_{15}$ & 1 \\
\hline & 9 & $S_{9}$ & $t_{4}, t_{13}, t_{17}$ & $t_{1}, t_{10}, t_{15}$ & 2 \\
\hline & 10 & $S_{10}$ & $t_{4}, t_{13}$ & $t_{1}, t_{9}$ & 1 \\
\hline
\end{tabular}

The controlled net after adding above changeable control specifications is illustrated in Figure 20. The place and arcs of the controller are illustrated with blue lines. 


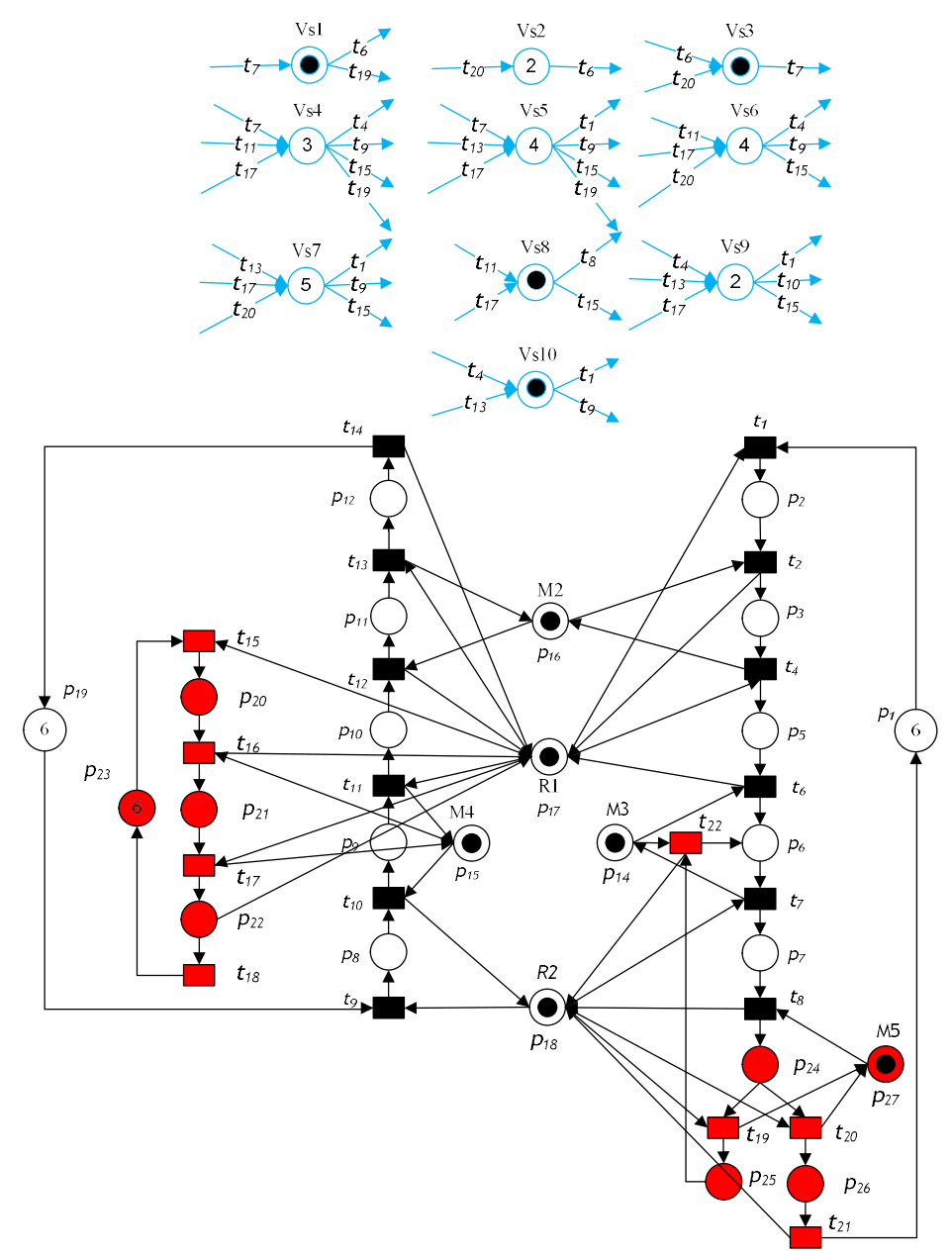

Figure 20. Controlled reconfigurable $S^{3} \mathrm{PR}$ net after adding changeable control specifications.

\section{Conclusions}

This paper develops a novel two-step solution for quick and accurate reconfiguration of supervisory controllers for deadlock control in RMSs with dynamic changes. In the first step, the net rewriting system is used to design a reconfigurable PN model under dynamic configurations. The obtained model guarantees boundedness behavioral property but may not guarantee the other properties of a Petri net model (i.e., liveness and reversibility). The second step proposes an automatic deadlock prevention policy for reconfigurable Petri net using the siphon control method based on a place invariant to solve the deadlock problem with dynamic structure changes in RMSs and guarantee the liveness and reversibility properties for the system. The proposed method is validated using the GPenSIM tool and compared with existing methods in the literature to highlight its ability of adapting to RMS configuration changes.

The major advantages of the developed approach are as follows: (1) It does not need to compute reachability graphs as illustrated in Algorithm 2, Section 3, and has low-overhead computation as proved in Theorems 10 and 11, Section 4.4. (2) It can automatically and dynamically modify the structure of a Petri net model without affecting its behavioral properties, i.e., liveness, boundedness, and reversibility as illustrated in Algorithm 2, Section 3. (3) It allows rapid reconfigurability and reusability of the controller during reconfiguration as shown in Algorithm 2, Section 3. (4) It can easily handle any dynamical changes in RMSs compared with the studies in Badouel et al. [39], Llorens and Oliver [34], Wu and Zhou [25], and Kaid et al. [7] as shown in Algorithm 2, Section 3. (5) The GPenSIM code is developed for designing, simulation, validation, and performance analysis of deadlock problems with dynamic structure changes in RMSs and the correctness of the proposed 
approach is proven and compared with the studies in Ezpeleta et al. [43], Li and Zhou [44], and Kaid et al. [6] as shown in Section 4.5. (6) Based on Theorems 10 and 11, the computational complexity of the proposed approach has polynomial time complexity. Therefore, it has low computational complexity and can be applicable to other types of complex systems such as mass customization manufacturing, lean productivity, agile manufacturing, and flexible manufacturing systems. (7) It can consider systems with sequential and complex resource requirements, meaning that a set of system resources can be used and shared to process each component according to sequential processes that depend on the step-by-step discrete execution and multiple processes that depend on the execution at the same time as shown in numerical example.

The limitation of the developed approach is that the obtained models lack an appropriate conversion approach from the PN model into control languages for application. Thus, our future research will examine the developed approach to have an automatic method to examine the applicability of the obtained models for real world manufacturing systems.

Author Contributions: Conceptualization, H.K. and A.A.-A.; software, H.K. and R.D.; resources, H.K., A.A.-A. and R.D.; formal analysis, H.K. and A.A.-A.; investigation, H.K., A.A.-A. and Z.L.; validation, H.K., A.A.-A., Z.L. and R.D.; writing —original draft preparation, H.K., A.A.-A. and Z.L.; writing-review and editing, H.K., A.A.-A., Z.L. and R.D.; visualization, H.K., A.A.-A. and Z.L.; supervision, A.A.-A., and Z.L. All authors have read and agreed to the published version of the manuscript.

Funding: This research was funded by King Saud University through Researchers Supporting Project Number (RSP-2020/62).

Acknowledgments: The authors would like to thank King Saud University for funding and supporting this research through Researchers Supporting Project Number (RSP-2020/62).

Conflicts of Interest: The authors declare no conflict of interest.

\section{References}

1. Hu, Y.; Ma, Z.; Li, Z. Design of Supervisors for Active Diagnosis in Discrete Event Systems. IEEE Trans. Autom. Control 2020. [CrossRef]

2. Wang, D.; Wang, X.; Li, Z. Nonblocking Supervisory Control of State-Tree Structures with Conditional-Preemption Matrices. IEEE Trans. Ind. Inform. 2019, 16, 3744-3766. [CrossRef]

3. Mehrabi, M.G.; Ulsoy, A.G.; Koren, Y. Reconfigurable manufacturing systems: Key to future manufacturing. J. Intell. Manuf. 2000, 11, 403-419. [CrossRef]

4. Katz, R. Design principles of reconfigurable machines. Int. J. Adv. Manuf. Technol. 2007, 34, 430-439. [CrossRef]

5. Patel, R.; Gojiya, A.; Deb, D. Failure Reconfiguration of Pumps in Two Reservoirs Connected to Overhead Tank. In Innovations in Infrastructure; Springer: Berlin/Heidelberg, Germany, 2019; pp. 81-92.

6. Kaid, H.; Al-Ahmari, A.; Li, Z.; Davidrajuh, R. Single controller-based colored Petri nets for deadlock control in automated manufacturing systems. Processes 2020, 8, 21. [CrossRef]

7. Kaid, H.; Al-Ahmari, A.; Li, Z.; Davidrajuh, R. Intelligent colored token Petri nets for modeling, control, and validation of dynamic changes in reconfigurable manufacturing systems. Processes 2020, 8, 358. [CrossRef]

8. Li, Z.; Zhou, M. Deadlock Resolution in Automated Manufacturing Systems: A Novel Petri Net Approach; Springer Science \& Business Media: Berlin/Heidelberg, Germany, 2009.

9. Zan, X.; Wu, Z.; Guo, C.; Yu, Z. A Pareto-based genetic algorithm for multi-objective scheduling of automated manufacturing systems. Adv. Mech. Eng. 2020, 12, 1687814019885294. [CrossRef]

10. Li, L.; Basile, F.; Li, Z. An approach to improve permissiveness of supervisors for GMECs in time Petri net systems. IEEE Trans. Autom. Control 2019, 65, 237-251. [CrossRef]

11. Liu, Y.; Cai, K.; Li, Z. On scalable supervisory control of multi-agent discrete-event systems. Automatica 2019, 108, 108460. [CrossRef]

12. Chen, Q.; Yin, L.; Wu, N.; El-Meligy, M.A.; Sharaf, M.A.F.; Li, Z. Diagnosability of vector discrete-event systems using predicates. IEEE Access 2019, 7, 147143-147155. [CrossRef] 
13. Kaid, H.; Al-Ahmari, A.; Nasr, E.A.; Al-Shayea, A.; Kamrani, A.K.; Noman, M.A.; Mahmoud, H.A. Petri Net Model Based on Neural Network for Deadlock Control and Fault Detection and Treatment in Automated Manufacturing Systems. IEEE Access 2020, 8, 103219-103235. [CrossRef]

14. Zhao, M. An integrated control method for designing non-blocking supervisors using Petri nets. Adv. Mech. Eng. 2017, 9, 1687814017700829. [CrossRef]

15. Chen, Y.; Li, Z.; Khalgui, M.; Mosbahi, O. Design of a maximally permissive liveness-enforcing Petri net supervisor for flexible manufacturing systems. Autom. Sci. Eng. IEEE Trans. 2011, 8, 374-393. [CrossRef]

16. Al-Ahmari, A.; Kaid, H.; Li, Z.; Davidrajuh, R. Strict Minimal Siphon-Based Colored Petri Net Supervisor Synthesis for Automated Manufacturing Systems with Unreliable Resources. IEEE Access 2020. [CrossRef]

17. Kaid, H.; Al-Ahmari, A.; El-Tamimi, A.M.; Abouel Nasr, E.; Li, Z. Design and implementation of deadlock control for automated manufacturing systems. South Afr. J. Ind. Eng. 2019, 30, 1-23. [CrossRef]

18. Chao, D.Y. Improvement of suboptimal siphon-and FBM-based control model of a well-known $S^{3} P R$. IEEE Trans. Autom. Sci. Eng. 2011, 8, 404-411. [CrossRef]

19. Ghaffari, A.; Rezg, N.; Xie, X. Design of a live and maximally permissive Petri net controller using the theory of regions. IEEE Trans. Robot. Autom. 2003, 19, 137-141. [CrossRef]

20. Uzam, M. The use of the Petri net reduction approach for an optimal deadlock prevention policy for flexible manufacturing systems. Int. J. Adv. Manuf. Technol. 2004, 23, 204-219. [CrossRef]

21. Sun, D.; Chen, Y.; El-Meligy, M.A.; Sharaf, M.A.F.; Wu, N.; Li, Z. On algebraic identification of critical states for deadlock control in automated manufacturing systems modeled with Petri nets. IEEE Access 2019, 7, 121332-121349. [CrossRef]

22. Nasr, E.A.; El-Tamimi, A.M.; Al-Ahmari, A.; Kaid, H. Comparison and Evaluation of Deadlock Prevention Methods for Different Size Automated Manufacturing Systems. Math. Probl. Eng. 2015, 501, 1-19. [CrossRef]

23. Lee, S.; Tilbury, D.M. Deadlock-free resource allocation control for a reconfigurable manufacturing system with serial and parallel configuration. IEEE Trans. Syst. ManCybern. Part C (Appl. Rev.) 2007, 37, 1373-1381. [CrossRef]

24. Li, J.; Dai, X.; Meng, Z. Automatic reconfiguration of petri net controllers for reconfigurable manufacturing systems with an improved net rewriting system-based approach. IEEE Trans. Autom. Sci. Eng. 2009, 6, 156-167. [CrossRef]

25. Wu, N.; Zhou, M. Intelligent token Petri nets for modelling and control of reconfigurable automated manufacturing systems with dynamical changes. Trans. Inst. Meas. Control 2011, 33, 9-29.

26. Sampath, R.; Darabi, H.; Buy, U.; Liu, J. Control reconfiguration of discrete event systems with dynamic control specifications. IEEE Trans. Autom. Sci. Eng. 2008, 5, 84-100. [CrossRef]

27. Dumitrache, I.; Caramihai, S.; Stanescu, A. Intelligent agent-based control systems in manufacturing. In Proceedings of the 2000 IEEE International Symposium on Intelligent Control. Held jointly with the 8th IEEE Mediterranean Conference on Control and Automation (Cat. No. 00CH37147), Rio Patras, Greece, 19-19 July 2000; pp. 369-374.

28. Ohashi, K.; Shin, K.G. Model-based control for reconfigurable manufacturing systems. In Proceedings of the 2001 ICRA. IEEE International Conference on Robotics and Automation (Cat. No. 01CH37164), Seoul, Korea, 21-26 May 2001; pp. 553-558.

29. Kalita, D.; Khargonekar, P.P. Formal verification for analysis and design of logic controllers for reconfigurable machining systems. IEEE Trans. Robot. Autom. 2002, 18, 463-474. [CrossRef]

30. Almeida, E.E.; Luntz, J.E.; Tilbury, D.M. Event-condition-action systems for reconfigurable logic control. IEEE Trans. Autom. Sci. Eng. 2007, 4, 167-181. [CrossRef]

31. Zhang, L.; Rodrigues, B. Modelling reconfigurable manufacturing systems with coloured timed Petri nets. Int. J. Prod. Res. 2009, 47, 4569-4591. [CrossRef]

32. Kahloul, L.; Bourekkache, S.; Djouani, K.; Chaoui, A.; Kazar, O. Using high level Petri nets in the modelling, simulation and verification of reconfigurable manufacturing systems. Int. J. Softw. Eng. Knowl. Eng. 2014, 24, 419-443. [CrossRef]

33. Yu, Z.; Guo, F.; Ouyang, J.; Zhou, L. Object-oriented Petri nets and $\pi$-calculus-based modeling and analysis of reconfigurable manufacturing systems. Adv. Mech. Eng. 2016, 8, 1687814016677698. [CrossRef]

34. Llorens, M.; Oliver, J. Structural and dynamic changes in concurrent systems: Reconfigurable Petri nets. IEEE Trans. Comput. 2004, 53, 1147-1158. [CrossRef] 
35. Li, J.; Dai, X.; Meng, Z. Improved net rewriting systems-based rapid reconfiguration of Petri net logic controllers. In Proceedings of the 31st Annual Conference of IEEE Industrial Electronics Society, Raleigh, NC, USA, 6-10 November 2005.

36. da Silva, R.M.; Benítez-Pina, I.F.; Blos, M.F.; Santos Filho, D.J.; Miyagi, P.E. Modeling of reconfigurable distributed manufacturing control systems. IFAC-Pap. 2015, 48, 1284-1289. [CrossRef]

37. Berthomieu, B.; Ribet, P.-O.; Vernadat, F. The tool TINA-construction of abstract state spaces for Petri nets and time Petri nets. Int. J. Prod. Res. 2004, 42, 2741-2756. [CrossRef]

38. Bonet, P.; Catalina, M.L.; Puigjaner, R. A Petri Net tool for performance modeling. In Proceedings of the 23rd Latin American Conference on Informatics (CLEI 2007), San Jose, Costa Rica, October 2007.

39. Badouel, E.; Llorens, M.; Oliver, J. Modeling concurrent systems: Reconfigurable nets. In Proceedings of the PDPTA, Las Vegas, NV, USA, 23-26 June 2003; pp. 1568-1574.

40. Yamalidou, K.; Moody, J.; Lemmon, M.; Antsaklis, P. Feedback control of Petri nets based on place invariants. Automatica 1996, 32, 15-28. [CrossRef]

41. Moody, J.O.; Antsaklis, P.J. Supervisory Control of Discrete Event Systems Using Petri Nets; Springer Science \& Business Media: Berlin/Heidelberg, Germany, 2012; Volume 8.

42. Davidrajuh, R. Modeling Discrete-Event Systems with GPenSIM: An Introduction; Springer: Berlin/Heidelberg, Germany, 2018.

43. Ezpeleta, J.; Colom, J.M.; Martinez, J. A Petri net based deadlock prevention policy for flexible manufacturing systems. IEEE Trans. Robot. Autom. 1995, 11, 173-184. [CrossRef]

44. Li, Z.; Zhou, M. Elementary siphons of Petri nets and their application to deadlock prevention in flexible manufacturing systems. IEEE Trans. Syst. Man Cybern. Part A Syst. Hum. 2004, 34, 38-51. [CrossRef]

45. Piroddi, L.; Cordone, R.; Fumagalli, I. Selective siphon control for deadlock prevention in Petri nets. IEEE Trans. Syst. Man Cybern. Part A: Syst. Hum. 2008, 38, 1337-1348. [CrossRef]

46. Chen, Y.; Li, Z.; Zhou, M. Behaviorally optimal and structurally simple liveness-enforcing supervisors of flexible manufacturing systems. IEEE Trans. Syst. Man Cybern. Part A Syst. Hum. 2012, 42, 615-629. [CrossRef]

(C) 2020 by the authors. Licensee MDPI, Basel, Switzerland. This article is an open access article distributed under the terms and conditions of the Creative Commons Attribution (CC BY) license (http://creativecommons.org/licenses/by/4.0/). 